\title{
Design and Psychometric Evaluation of American Sign Language Translations of Usability Questionnaires
}

\author{
LARWAN BERKE, MATT HUENERFAUTH, and KASMIRA PATEL, \\ Rochester Institute of Technology
}

\begin{abstract}
To promote greater inclusion of people who are Deaf and Hard of Hearing (DHH) in studies conducted by Human-Computer Interaction (HCI) researchers or professionals, we have undertaken a project to formally translate several standardized usability questionnaires from English to ASL. Many deaf adults in the U.S. have lower levels of English reading literacy, but there are currently no standardized usability questionnaires available in American Sign Language (ASL) for these users. A critical concern in conducting such a translation is to ensure that the meaning of the original question items has been preserved during translation, as well as other key psychometric properties of the instrument, including internal reliability, criterion validity, and construct validity. After identifying best-practices for such a translation and evaluation project, a bilingual team of domain experts (including native ASL signers who are members of the Deaf community) translated the System Usability Scale (SUS) and Net Promoter Score (NPS) instruments into ASL and then conducted back-translation evaluations to assess the faithfulness of the translation. The new ASL instruments were employed in usability tests with DHH participants, to assemble a dataset of response scores, in support of the psychometric validation. We are disseminating these translated instruments, as well as collected response values from DHH participants, to encourage greater participation in HCI studies among DHH users.
\end{abstract}

CCS Concepts: • Human-centered computing $\rightarrow$ Empirical studies in HCI; Accessibility design and evaluation methods;

Additional Key Words and Phrases: System usability scale, SUS, net promoter score, NPS, American Sign Language, translation, ASL-SUS, ASL-NPS, criterion validity, internal reliability, construct validity, factor analysis

ACM Reference format:

Larwan Berke, Matt Huenerfauth, and Kasmira Patel. 2019. Design and Psychometric Evaluation of American Sign Language Translations of Usability Questionnaires. ACM Trans. Access. Comput. 12, 2, Article 6 (June 2019), 43 pages.

https://doi.org/10.1145/3314205

This material is based upon work supported by the National Science Foundation under Awards No. 1400802 and No. 1462280, by the Department of Health and Human Services under Award No. 90DPCP0002-01-00, by a Google Faculty Research Award, and by the National Technical Institute for the Deaf (NTID). Furthermore, this material is based upon work supported by the National Science Foundation Graduate Research Fellowship under Grant No. 1746056. Any opinions, findings, and conclusions or recommendations expressed in this material are those of the authors and do not necessarily reflect the views of the National Science Foundation.

Authors' addresses: L. Berke, M. Huenerfauth, and K. Patel, Rochester Institute of Technology, 152 Lomb Memorial Drive, Rochester, NY, 14618; emails: larwan.berke@mail.rit.edu, matt.huenerfauth@rit.edu, kasmira.patel4@gmail.com.

Permission to make digital or hard copies of all or part of this work for personal or classroom use is granted without fee provided that copies are not made or distributed for profit or commercial advantage and that copies bear this notice and the full citation on the first page. Copyrights for components of this work owned by others than ACM must be honored. Abstracting with credit is permitted. To copy otherwise, or republish, to post on servers or to redistribute to lists, requires prior specific permission and/or a fee. Request permissions from permissions@acm.org.

(C) 2019 Association for Computing Machinery.

1936-7228/2019/06-ART6 \$15.00

https://doi.org/10.1145/3314205 


\section{INTRODUCTION}

$\mathrm{HCI}$ researchers or designers often gather feedback from study participants by asking them to respond to questions about their impression of a system, and many studies will use pre-existing questionnaires for this purpose. To ensure that technology is accessible to diverse users, researchers and designers should ideally include people with disabilities during evaluation studies: The focus of our research is on finding ways to make it easier for researchers to include people who are Deaf or Hard-of-Hearing (DHH) in such studies. One logistical challenge that designers face when including DHH users in empirical studies is that many people who are DHH prefer to communicate using a sign language, such as American Sign Language (ASL). In fact, studies indicate that there are a half-million people who consider ASL as a primary means of communication [50] in the U.S. Previous research on English literacy among U.S. deaf adults has found that many have lower literacy than their hearing peers [78]. Furthermore, there has been prior research into the perils of administering English-based questionnaires to non-native users [24]. Researchers have found that participants who were presented with questionnaire instruments not in their native language may not fully understand the questions, which may lead to discomfort or responses that are less reliable [26]. Thus, it is imperative to undertake a translation effort to produce ASL versions of such instruments, for members of the Deaf community who prefer communication in ASL.

Our team decided to pick two survey instruments to translate in this project: the System Usability Scale (SUS) and the Net Promoter Score (NPS)-more details appear in Section 2.2. SUS has been used to evaluate a wide range of products, including hardware, computer software, websites, and mobile applications. Given the ease with which it is administered and scored, it has become a ubiquitous measurement instrument in HCI research and in commercial HCI usability testing. NPS is being used extensively in the field of marketing research, and has been shown to be a quick method to gauge reactions from participants during studies, due to the simplicity of administering only one question item. An additional rationale for focusing on these instruments was that SUS and NPS are freely available-see Section 2.3 for a literature review of prior work on translating SUS, which motivated the methodology for our project.

We therefore investigate how to translate standardized usability questionnaires, into ASL. By making this translated instrument (in the form of ASL videos with translated instructions and question items) available to the HCI research community, our goal is to enable researchers to more easily include DHH participants in their studies, alongside hearing participants. In addition to creating this ASL translation, we have also conducted an evaluation to determine whether it preserves the meaning and other characteristics of the original English version, to enable researchers to compare scores across both versions.

Finally, it is the intent of our team to validate the English-to-ASL translation methodology used in this project as the basis for our future translation efforts of additional usability survey instruments.

\subsection{An Extension of Prior Research Efforts}

This article is an extended version of a paper originally presented at the 2017 ACM SIGACCESS Conference on Computers and Accessibility (ASSETS'17) [31]. In that conference paper, we presented our initial work on translating the SUS (described in Section 4), during which we developed the translation process and evaluated the SUS questionnaire with DHH participants. While this conference paper described our process and reported on our evaluation of the translated instrument (indicating that the translation had preserved several important characteristics of the original instrument), we had identified some limitations in our initial evaluation and received feedback from reviewers and attendees at the conference. The new contributions of this article are as follows: 
We conducted additional follow-up studies that increased the size of the dataset of participant responses from the prior work, and this larger dataset enabled us to conduct additional analyses of the ASL-SUS (including a factor analysis of the instrument). Finally, we repeated this entire process of translation and evaluation for another survey instrument: the NPS (described in Section 5). With the greater page length of this article, we are also able to provide more detail about the translation, our user studies, and the psychometric evaluation we conducted.

\subsection{Structure of This Article}

This article is organized as follows: Section 2 surveys prior work on HCI research with DHH users, standardized questionnaires, and prior efforts to translate them into other languages. Sections 3 and 3.1 outline our research questions and methodology for creating and evaluating the ASL version. Section 4 describes in detail the ASL translation of SUS, and Section 5 repeats the same process but for the NPS instrument. Section 6 summarizes our conclusions and future work.

\section{LITERATURE SURVEY}

\subsection{Technology Research with DHH Users}

While it is possible to identify examples of published research in which people with disabilities were included a study, it is much more difficult to enumerate examples of studies in which people with disabilities were excluded from usability studies or research. Many researchers operate with the assumption that the logistical barriers to inclusion are too great, especially when the study's focus is on the general population rather than on people with disabilities specifically. As discussed in Reference [68], while there is general consensus among HCI researchers that the participants in a study should be representative of the population of users, there can be a variety of challenges in recruiting participants with disabilities. People with disabilities are often not included in user testing, which can lead to inaccurate results [68]. In fact, a popular research-methods textbook for training future $\mathrm{HCI}$ professionals devotes an entire chapter to motivating students that people with disabilities should be included in studies and explaining how to overcome logistical barriers to doing so [38]. Rather than recruit users with disabilities, some researchers include proxy users, e.g. sighted users wearing blindfolds or hearing users with the computer muted $[38,68]$. Others have examined the use of remote usability testing for people with disabilities, which addresses some barriers to recruitment due to population sparsity or difficulty traveling, but remote testing but does not address the English literacy issues of many DHH users (discussed above), unless steps are taken to provide question items using ASL videos, e.g., as in References [67, 77].

When publishing their research at competitive research conferences or journals in the field of computing accessibility, researchers will generally include people with disabilities in their empirical studies [68]. For instance, researchers studying technologies related to sign language animation or recognition often conduct evaluation studies with participants who are $\mathrm{DHH}[20,32]$. In addition, technology researchers studying the current barriers faced by people who are $\mathrm{DHH}$ also tend to include DHH participants in studies: For instance, researchers have examined the communication challenges of DHH users in business or educational contexts [17, 33, 76].

In much of this aforementioned research with DHH users, researchers have used nonstandardized survey questions, often created specifically for each particular project. In some cases, these question items are provided in the form of ASL videos, which may be displayed alongside English text versions of the question items. For instance, a recent online survey of DHH students, to study their communication approaches used during meetings with hearing peers, included both English and ASL versions of each question [76]. Another online survey used ASL videos to present question items, to collect judgments from ASL signers about the intelligibility of ASL videos of various levels of quality [77]. 
Some prior accessibility researchers have created and disseminated standardized survey instruments in the form of ASL videos. In Reference [32], researchers sought to identify relationships between DHH participants' opinions about ASL animation technology and their responses to standardized surveys about technology use. In support of this research, the authors created ASL translations of pre-existing English survey questions about technology use (originally designed for a survey targeted at older users) and displayed these items to their participants in the form of ASL videos. However, the authors did not conduct a formal assessment of the quality of their ASL translations. In References [30,32], researchers disseminated sets of question items (in the form of both English text and ASL video) that could be used to evaluate the quality of ASL animation generation systems, to promote a standardized set of evaluation instruments among that research community, to enable comparisons of results across studies. However, none of this prior research produced translations of commonly used HCI usability survey instruments, nor did these prior studies include a formal assessment of translation quality.

A key premise of our work is that if there were greater availability of question items (available as both English text and ASL videos) suitable for evaluating usability of software or websites, more HCI researchers may include DHH participants in their studies, even when the study is not specifically focused on DHH users.

\subsection{Standardized Usability Questionnaires and Psychometric Properties}

When conducting a study to measure participants' subjective opinion about the usability of some technology, HCI researchers often use standardized questionnaires, i.e., official, accepted versions of questionnaires with a commonly agreed-upon set of questions, presented in a particular order. These standardized instruments typically include rubrics for how to calculate a final score based on a participant's responses. In this way, when multiple researchers make use of the same questionnaire, it is easier to compare results across studies. Further, it is possible to archive participant scores across a large number of studies, to create a historical dataset of response scores, perhaps subcategorized as to the type of technology that had been evaluated in each study. This set of published response values (or tables containing mean and standard deviations) is referred to as "norms," and the existence of this large quantity of historical data makes it easier to evaluate the results of a new study. That is, after a researcher has used a standardized instrument to produce a score representing the usability of some technology, they can compare their participants' responses to these norms to understand how their new results compare, in general, to prior studies.

2.2.1 List of Standardized Instruments. There are a variety of standardized instruments used in the field of HCI for usability testing, and we outline a selection of these instruments below. For a listing of many additional instruments, the reader may consult [66]:

- System Usability Scale (SUS): An extremely popular instrument, and the primary focus of this article, is the SUS, which consists of a ten-item Likert scale with English statements about the usability of some product [10]. For each item, participants indicate their agreement on a five-point scale from "Strongly Disagree" to "Strongly Agree." Based on the participants' responses, researchers use a rubric to calculate a final score on a range from 0 to 100 , with higher scores indicating that the participant believed the product to be very usable.

- Computer System Usability Questionnaire (CSUQ): Another widely-used instrument is the CSUQ, a 19-item survey utilizing a 7-point scale with " 1 = Strongly Disagree" and "7 = Strongly Agree" for evaluating systems [40]. The CSUQ evolved from the Post-Study System Usability Questionnaire (PSSUQ), a survey developed by the same research group 
at IBM [41]. One notable difference between the SUS and the CSUQ is the addition of open-ended response entries after each question item, which allow participants to further explain their answers by describing their experience with the system.

- Usability Metric for User Experience (UMUX): The UMUX is another popular instrument, utilizing a 4-item Likert scale (7-point) to assess a system's usability [25]. The design of UMUX was partially motivated as an attempt to address some some limitations of SUS, such as using a 5-point scale (which some researchers believed to be inadequate in some cases) and word choices in some SUS question items that confounded non-native speakers of English. There is also a shortened version of the UMUX, the UMUX-LITE [44], which contains only two question items.

- After-Scenario Questionnaire (ASQ): The ASQ was developed to evaluate how participants gauged their performance after completing a set of tasks for a given system [39]. The ASQ asks participants three 7-point Likert questions, with an additional answer choice added to the scale: "not applicable (N/A)." The three items asked participants about the ease of completion, amount of time, and the support provided for the tasks they undertook during experiments.

- Net Promoter Score (NPS): Finally, the NPS is an extremely popular and brief instrument, which is often used in contexts beyond traditional usability testing of products, e.g., measuring a respondent's satisfaction with a customer-service experience with a business. NPS is a single-item questionnaire that asks participants to report their likelihood of recommending a product (or company) to other people [53], on a scale ranging from 0 to 10 , where 0 means "Not at all likely" and 10 means "Extremely likely." Rather than simply reporting average scores across a set of participants, practitioners utilizing NPS typically calculate the final score by subtracting the percentage of participants whom selected 0 to 6 (detractors) from the percentage of participants whom selected 9 or 10 (promoters).

2.2.2 Desirable Psychometric Properties. Typically, the creator of a standardized questionnaire selects a subset of questions, from an initially larger pool of questions, through a process of psychometric validation. The goal is to create a measurement instrument with a set of desirable psychometric properties $[40,66]$. Since we discuss some of these properties in this article, for the reader's convenience, we provide brief definitions below for how we use some terms. Detailed definitions appear in References [3, 40, 66].

- Internal Reliability refers to its internal consistency, i.e., whether all of the items in the instrument contribute equally to its score; this property is often assessed by calculating the Cronbach's Alpha coefficient for the group of items [16].

- Criterion Validity refers to whether the instrument relates to some other external property that can be measured; this property is often assessed based on whether the resulting score is correlated with some other trusted measure that respondents complete concurrently (i.e., another instrument they complete at the same time) or to its ability to predict some outcome.

- Construct Validity refers to whether the instrument actually measures the real-world phenomena it is meant to, e.g., usability; this property is often assessed by examining the internal factor structure, i.e., whether subsets of questions on the questionnaire are correlated and whether these clusters relates to specific sub-scores or factors to be measured.

- Content Validity refers to whether the questionnaire covers all of the essential aspects of the real-world phenomena it is supposed to measure; this property is often assessed by asking a panel of expert judges to evaluate the individual items. 


\subsection{Translating Surveys to Other Languages}

Prior research has established that asking participants to respond to a questionnaire in their nonnative language affects their response scores [24, 26]. For this reason, researchers have established customary methods for translating a standardized survey into another language and evaluating whether the meaning of the original was preserved [26]. Further, researchers who translate a standardized questionnaire often evaluate the new version to determine whether certain desirable psychometric properties of the original (Section 2.2.2) were preserved during the translation process. This section describes prior work in translating standardized usability questionnaires to other languages and the methods used to evaluate the success of those efforts.

In translation projects it is often useful to consider the scope of the translation and there are two aspects (language and modality) to consider, with several possible combinations:

- Same language, different modality (e.g., spoken English to written English)

- Different language, same modality (e.g., written English to written Portuguese)

- Different language, different modality (e.g., written English to ASL)

In most of the prior work described below, the translation falls into the category of "different language, same modality," in that the authors were translating a survey instrument from one written language to another. In the case of our project, we are seeking to translate survey instruments from English into ASL, which would fall into the category of "different language, different modality."

2.3.1 Prior Translation Efforts. Our literature review found many papers that translated HCI questionnaires such as SUS [2, 8, 9, 19, 23, 45, 47, 60, 70, 71], and below we focus on a few typical examples of this methodology:

- English-to-Persian SUS: Researchers in Iran translated the SUS into Persian, with a goal of creating an Iranian version that retained the psychometric properties of the original [19]. To ensure that the content of the new instrument was valid, a panel of 10 experts first analyzed the Persian version of the questions. Next, the authors conducted a study with 202 university students to evaluate the resulting survey scores.

- English-to-Slovene SUS: Researchers translated SUS into Slovene [8]. To evaluate the translation, the authors assembled a committee of reviewers with multidisciplinary backgrounds. The authors also evaluated their new instrument in a user study with 182 participants.

- English-to-Turkish CSUQ: Researchers translated the CSUQ from English into Turkish [23]. While we do not conduct a translation of CSUQ in this article, this metric is a similar standardized usability questionnaire to SUS; so, the process by which researchers translated and evaluated it is relevant for us to consider. After conducting their translation, the authors evaluated the validity of the Turkish version by using the instrument in a user study with 97 participants to evaluate its psychometric properties.

- English-to-German SUS: Researchers authored a German translation of SUS based, in part, on an online crowdsourcing effort by a large group of volunteers [45, 60]. However, no formal evaluation was conducted of the quality of the resulting translation nor of its psychometric properties.

- English-to-Polish SUS, CSUQ, and NPS: Researchers translated the SUS to Polish [9] and, in the same project, translated the CSUQ and NPS questionnaires. This work provides a useful demonstration of how researchers translating multiple instruments may calculate correlation between several usability metrics, which is an approach we have employed in our English-to-ASL translation of SUS and NPS in this article. 
- English-to-Portuguese SUS: Researchers translated the SUS into Portuguese, and analyzed whether the translation maintained the construct validity and internal reliability psychometric properties [47]. After evaluating their translation with 30 participants, the authors ensured that the construct validity was preserved by analyzing the correlation of the Portuguese SUS to the PSSUQ and to the ASQ.

In the literature review below, we analyze these prior efforts to translate standardized usability surveys into other languages along several dimensions, which correspond to the three subsections below. Throughout this literature review, key methodological elements of these prior studies are highlighted in bold font, and the research methodology for our translation of the questionnaires from English-to-ASL (Section 3.1) incorporates many key elements of the methodology of these prior translation projects.

2.3.2 Who Translated the Questionnaire, and How? The methods in prior projects to translate usability questionnaires have varied. In some cases, a team of experts have performed the translation, often as part of a multi-disciplinary team.

For example, in the English-to-Persian SUS project [19], an ergonomics specialist conducted the initial translation. In the English-to-Turkish CSUQ project, a multidisciplinary research group of language professionals and reviewers were responsible for the translation. The group consisted of five native Turkish reviewers, two were bilingual reviewers, a native Turkish language expert and also usability expert who was responsible for coordinating the translation effort [23]. In References $[45,60]$, a group of volunteers suggested through online crowdsourcing how to translate the questions into German. An advantage of this approach is that it reduces the cost for researchers in conducting the translation, and it also provides a method by which questions could be translated into a wide variety of languages. However, the quality must be assessed, e.g., researchers in References $[45,60]$ had to edit the final version to improve the translation quality, subsequent to the crowdsourcing.

Sections 4.1 and 5.1 will discuss how, in our project, we have asked a small team of experts to translate SUS and NPS into ASL.

2.3.3 How was the Translation Evaluated to Determine If the Original Meaning was Preserved? There are several ways that the quality of the translation can be evaluated to determine whether it is fluent and whether it has preserved the meaning of the original English version. In some projects, the team responsible for producing the translation conducts multiple rounds of revision, sometimes interleaved with rounds of user testing.

For instance, in the English-to-Persian SUS project [19], researchers conducted a study with 30 participants to identify linguistic problems in the translation; this pilot study led to the identification and integration of amendments into the final Persian SUS. In the English-to-Slovene SUS project [8], the translation process involved ten reviewers from the computer and natural sciences fields and three independent translators who were native Slovene speakers also fluent in English. The translation process was carried out in multiple stages, with members of the translation team considering comments from evaluators after each round. In the English-to-Turkish CSUQ project [23], the quality of the translation was evaluated through a committee review process. After a draft of the translation to Turkish was developed from the original, the draft underwent a threestage review process. This was aimed at modifying the Turkish version to preserve the meaning of the original English questions. Different participants were involved in each of the three stages. The nine members of the translation team independently evaluated the results after each round of testing and adjusted their translation to ensure that the meaning of the original questions were preserved. Each member of the research group reviewed the original and the revised versions of 
the Turkish SUS independently [23]. In the German-to-English SUS project [45, 60], researchers edited the output of the crowdsourced translations written by volunteers online.

In some projects, a back-translation procedure is used to identify problems in the translated text: In this approach, after the team has produced their initial forward translation of a questionnaire from language "A" to language "B," then the researchers set up an evaluation study in which some new group of people translate each question back into the original language $\mathrm{A}$. By comparing the original version of the survey $(\mathrm{A})$ to this back-translated version (A-to-B-to-A), the researchers can determine if some meaning or concepts were lost during the original A-to-B translation stage.

For example, in the English-to-Persian SUS project [19], two professional translators, who had lived in English-speaking countries, performed a back-translation after the original forward translation (English to Persian) had been carried out by the ergonomic specialist on the translation team. In the English-to-Slovene SUS project [8], the final round of revision included a back-translation procedure to look for any missing concepts.

Sections 4.3 and 5.3 will discuss how, in our project, we have decided to use a translation process that incorporates both: multiple rounds of revision along with a back-translation procedure to identify any translated items that fail to preserve the meaning of the original.

\subsubsection{How was the New Version of the Questionnaire Evaluated to Determine Whether It Pre-} served the Useful Psychometric Properties of the Original? In addition to evaluating the text itself, researchers have evaluated whether key statistical characteristics of the questionnaire were preserved after the translation process.

For example, in the English-to-Persian SUS project [19], a qualitative evaluation was also conducted involving a panel of ten experts to assess the content validity of the new instrument. Feedback from the expert group led to a revision of the translation of some items, then the researchers assessed their instrument through a study with 201 university students who evaluated a university food reservation system. The psychometric measures that were assessed based on these results included construct validity and internal reliability [19]. In the English-to-Slovene SUS project [8], a study involving 182 respondents (114 males, 86 females) was carried out using the new Slovene SUS to evaluate the usability of the Google Gmail website. The Slovene researchers evaluated their newly translated instrument's internal reliability, criterion validity, and construct validity. In the English-to-Turkish CSUQ project [23], the researchers evaluated their translated version by using it in a usability study. Since CSUQ has a factor structure (i.e., it consists of several subscales, each based on a subset of questions), the researchers evaluated its construct validity by examining whether scores for clusters of items in the newly translated version were appropriately correlated. The authors of the English-to-Portuguese SUS [47] used the Intraclass Correlation Coefficient (ICC) to validate their translation's reliability property; however, they reported a low ICC value and speculated that it was due to the SUS containing alternating "positive" and "negative" question items, which confounded their participants. While there is an "all-positive" version of the SUS [65], our team chose to not use that version in this project as we wanted to compare our data across a wide range of published research on the original SUS-we will report the ICC values regardless.

Sections 4.4 and 5.4 describes how we conducted a usability test with DHH participants responding to our new ASL version of the usability metrics; we assessed internal reliability of our items using Cronbach's Alpha [16]. We also asked participants to complete two additional usability questionnaires: (Adjective Scale), which had previously been used in Reference [5], and the NPS, to assess the criterion validity of our ASL translations. Finally, to evaluate the construct validity, we will conduct factor analysis on the ASL-SUS responses. However, since the NPS contains only one question item, it is not possible to do the same for that instrument. 


\subsection{Translating Surveys to ASL}

Prior researchers have translated English surveys into ASL for health-related studies; although they had a focus on medical issues, these researchers' methodologies are also relevant to our work.

Health researchers have translated the Behavioral Risk Factor Surveillance System survey (a standardized survey conducted across the U.S.) into ASL using a team of experts consisting of bilingual individuals, with a mix of Deaf community members and health researchers [27]. The team strove for ASL with meaning equivalence rather than word-for-word translation and for ASL that would be understandable to a wide set of deaf individuals [27]. After the team produced an ASL script (a text file containing sequences of English words representing sequences of ASL signs, in appropriate ASL word order), a researcher not on the translation team backtranslated the material to English. These were compared to the original English questions; in some cases, the translation team edited their ASL translations based on the results of this backtranslation evaluation [27]. Finally, native ASL signers (people who grew up using ASL since early childhood) performed the ASL versions while being video recorded; an additional native ASL signer "coach" sat behind the camera, watching the script, to ensure that the performer followed the script that had been produced by the translation team.

Other researchers have translated the Multidimensional Health Locus of Control survey from English into ASL [62]. They convened a focus group of both bilingual members of the Deaf community who were native ASL signers and ASL interpreters. This first focus group produced an ASL translation for each English question item. Next, the researchers convened a second focus group (with similar membership composition) to produce an English back-translation for each ASL item; this second focus group also evaluated how close their English back-translation was to the original English version of the question items. In cases of divergence, the second focus group was asked to recommend a modification to the ASL version to better preserve the meaning of the original. During the back-translation evaluation, there were many cases (15 of 24) in which back-translation lacked word-for-word equivalence with the original English item, yet the focus group determined that the meaning was sufficiently preserved [62].

While cross-cultural aspects are often a consideration during the translation from one language to another, it is important to note that in the case of translating materials originally written in English into ASL, there are also cross-cultural challenges, even though the target audience may be within the same country (e.g., the U.S., as in the case of the aforementioned health surveys). Various researchers, e.g., [36], have articulated cultural differences between U.S. Deaf community and the surrounding hearing majority population. Customarily, authors will user lower-case "deaf" and upper-case "Deaf" to distinguish between the audiological experience and this cultural community, respectively. ${ }^{1}$ This cultural aspect underscores the importance of not only having ASL experts on the translation team, but to also have individuals who are part of Deaf culture (e.g., born to Deaf parents, grew up with Deaf friends, attended schools for the Deaf, etc.). Those Deaf members of a translation team have an important role:

- making sure that the translation could be understood by this community through appropriate use of idioms or cultural context

\footnotetext{
${ }^{1}$ This distinction has been characterized as: "The lowercase 'deaf' refers to those for whom deafness is primarily an audiological experience. It is mainly used to describe those who lost some or all of their hearing in early or late life and who do not usually wish to have contact with signing Deaf communities, preferring to try and retain their membership of the majority society in which they were socialised. 'Deaf' refers to those born Deaf or deafened in early (sometimes late) childhood, for whom the sign languages, communities, and cultures of the Deaf collective represents their primary experience and allegiance, many of whom perceive their experience as essentially akin to other language minorities." [36, p. Glossary]
} 
- utilizing the full range of linguistic features in ASL (e.g., facial expressions, body movements, etc.) rather than English-influenced signing communication, which may utilize primarily the manual articulators (i.e., hand and arm movements)

- using their sophisticated ASL skill to gauge the fluency of the ASL translation (e.g., appropriate signing speed and pauses between clauses, etc.)

- ensuring that the chosen signs will be understood by all members of the Deaf community (since there are regional variations in the production of signs, similar to dialects in spoken languages)

- judging whether the translation could be understood by members of the Deaf community of varying English literacy levels (since problems may arise if English-influenced structures or word-choice are used in the ASL translation)

Sections 4.1 and 5.1 will discuss how, in our project, the team of experts conducting the translation included native ASL signers whom were part of the Deaf community. During translation, we created an ASL script, and during recording, a coach sat behind the camera while a native signer was recorded ${ }^{2}$. During our evaluation, a group of ASL interpreters produced the backtranslations, and when evaluating them, we considered meaning equivalence rather than word-for-word translation.

\section{RESEARCH QUESTIONS \& METHODS}

The goal of this project was to translate two commonly used standardized usability questionnaires: the SUS and NPS, from English into ASL, to make them suitable for use with DHH respondents who prefer to communicate using ASL. Our challenge was to preserve the meaning of the questions and other desirable characteristics (Section 2.2.2) so that results from our ASL instrument are comparable to results on the original English version. Furthermore, as in prior work (described in Section 4.4.2), we also translated the adjective scale instrument (abbreviated herein as "Adj") created by Reference [5], so that we could administer this additional instrument in some studies, to enable our analysis of the criterion validity of our ASL translation of SUS. For convenience, we refer to our translated versions as "ASL-SUS," "ASL-Adj," and "ASL-NPS." In the remainder of this article, we examine the following questions:

RQ1: Do the items in the ASL translation (of SUS and of NPS) preserve the meaning of the original English items, as measured through a back-translation study, in which ASL interpreters produce English back-translations and the meaning equivalence is compared to the original items?

RQ2: Deployed as the evaluation metric in a user study with DHH participants evaluating an artifact, does the ASL translation possess several key psychometric properties listed below?

(a) Internal Reliability, as measured by Cronbach's Alpha [16], indicating correlation among the set of question items.

(b) Criterion Validity, as measured by comparing the correlation between ASL-SUS and alternative measurement instruments collected concurrently from users. (We analyze the criterion validity of ASL-SUS through comparison with ASL-Adj, and ASL-NPS through comparison to ASL-SUS.)

\footnotetext{
${ }^{2}$ Although there has been research by our laboratory and other groups internationally on the use of computer-generated animations of sign language [22, 29, 32], the clearest use-case for such technology is for settings in which context must be frequently updated, as discussed in [1]. For a survey translation project in which a high-quality and understandable message is needed, with maximum fluency, we have therefore made use of videos of a native signer, following the recommendation in [82].
} 
(c) Construct Validity, as measured by analyzing the factor structure of the ASL-SUS, and comparing the resulting structure with prior published work [42]. (We are unable to do the same for ASL-NPS, since the NPS contains only a single question item.)

\subsection{Research Methods}

As mentioned in the introduction (Section 1.1), this article presents our efforts to translate two different standardized usability instruments: SUS and NPS. To provide a "big picture" of our methodology, Figure 1 (on the next page) enumerates the various stages in the translation of SUS and of NPS, with the activities for each instrument appearing as a long vertical column (SUS on the left, NPS on the right) and with the various stages of activity proceeding downward for each chronologically. Note: please consult Appendix A for a textual description of Figure 1.

3.1.1 Discussion of Figure 1 (Table of Contents Flowchart). Our translation project included five stages: The first was the translation of the English question items into ASL. In stage two, a native ASL signer produced video recordings of the items, and the third stage consisted of backtranslation and revision of the ASL videos. The fourth stage consisted of user studies with DHH users responding to the ASL version of the question items, to evaluate the quality of the translation and to assess the psychometric properties mentioned in RQ2 above. Finally, in stage five, we disseminate the ASL translation to the research community.

In Section 4, as we describe the translation and evaluation process for the ASL-SUS, we will explain each of these five stages in detail. Since our creation of the ASL-NPS used a similar sequence of methods, the description found in Section 5 will be briefer: Primarily noting the differences (if any) and focus on the results.

As discussed in Section 1.1, this article is an extension of work initially presented in a conference paper [31]. Figure 1 also highlights the novel elements of the research, as presented in this article: Specifically, the entire right side of this figure represents novel contributions of this article, as well as the additional data collected in two studies and used as the basis for additional psychometric analysis of the ASL version of SUS (as illustrated in the lower portion of the "Stage 4" box for the SUS).

Finally, Figure 1 also highlights how the various studies conducted with users have contributed to each stage of the project. For instance, during the evaluation of the quality of a translation of an instrument, it is common to conduct back-translation studies in which skilled translators or bilinguals attempt to "reverse" the translated instrument back into the original language, thereby enabling the research to compare it to the original, to look for information "lost in translation." Two such "back translation" studies were conducted, for each SUS and NPS instrument. Additionally, user studies were conducted with DHH participants using some technology artifact(s) and responding to the original (English) or new (ASL) version of the instrument.

3.1.2 Studies in This Project. Some usability studies had been reported in our prior conference paper ("2017-US-ASL" and "2017-US-ENG"), and this data had been used as the basis for an initial evaluation of our ASL translation of SUS. However, there was a limitation in that initial evaluation: While an in-person evaluation study with $30 \mathrm{DHH}$ users is reasonable in size, given comparable prior published accessibility studies with DHH users [30,32], some researchers have recommended $[23,51]$ recruiting a total number of participants equal to five times the number of items on an instrument that is being psychometrically validated, i.e., 50 participants in the case of SUS. Another limitation was our studies used to collect participant responses with SUS (studies "2017-US-ASL" and "2017-US-ENG") only asked the participants to evaluate a single artifact (a university website, as described in Section 4.4). For this reason, in our new user studies ("2018-US-ASL" and "2018-USENG”), we asked users to evaluate several technology items and respond to the usability instrument 


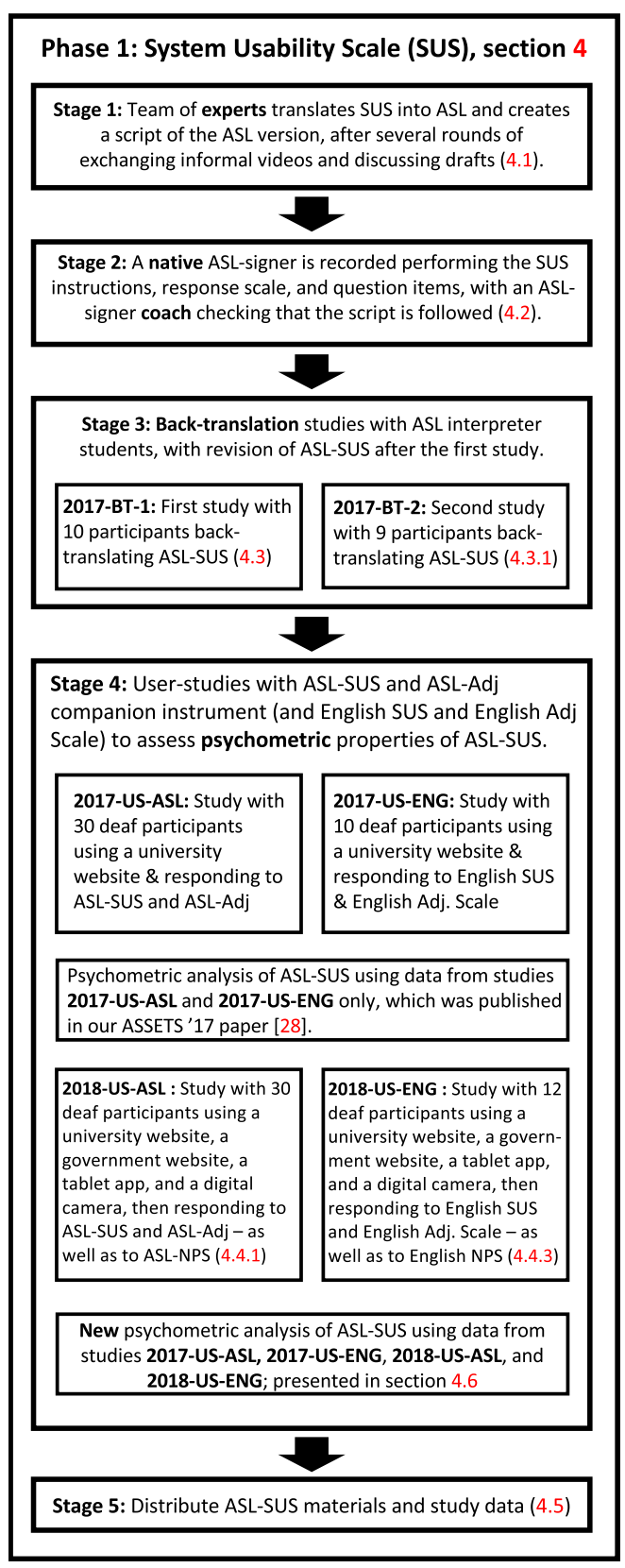

Phase 2: Net Promoter Score (NPS), section 5

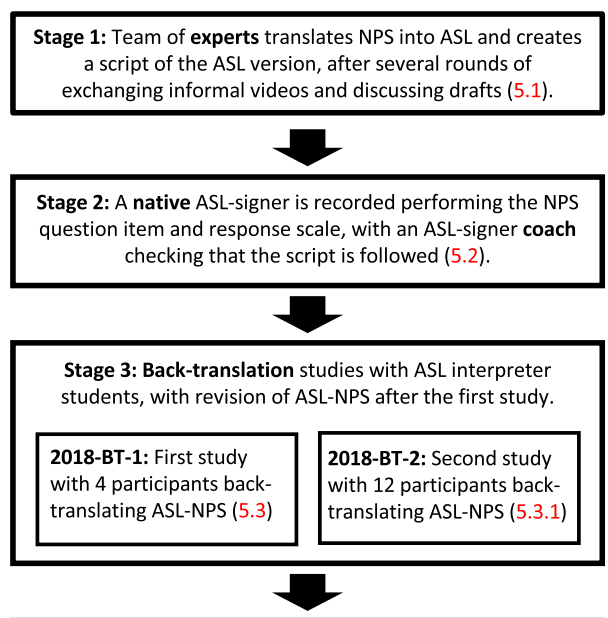

Stage 4: User-studies with ASL-NPS (and English NPS, and with use of ASL-SUS as a comparison instrument) to assess psychometric properties of the new ASL-NPS instrument. In addition, these two studies also included ASL or English SUS, in order to accumulate more response data for the SUS instrument in Phase 1, to enable a new psychometric analysis to be performed for ASL-SUS, with a greater pool of data (discussed in section 4.4).

\begin{tabular}{|l|l|l|}
\hline $\begin{array}{l}\text { 2018-US-ASL: Study with } 30 \\
\text { deaf participants using a } \\
\text { university website, a } \\
\text { government website, a } \\
\text { tablet app, and a digital } \\
\text { camera, then responding to } \\
\text { ASL-NPS - as well as to ASL- } \\
\text { SUS and ASL-Adj (4.4.1) }\end{array}$ & $\begin{array}{l}\text { 2018-US-ENG: Study with } \\
12 \text { deaf participants using } \\
\text { a university website, a } \\
\text { government website, a } \\
\text { tablet app, and a digital } \\
\text { camera, then responding } \\
\text { to English NPS - as well as } \\
\text { to English SUS, and English } \\
\text { Adj. Scale (4.4.3) }\end{array}$ \\
\hline
\end{tabular}

Note: The data from studies 2018-US-ASL and 2018-USENG were used to assess the psychometric properties of ASL-NPS (see below), and to supplement the response data collected for the ASL-SUS (from Phase 1: studies 2017-US-ASL and 2017-US-ENG), to enable a larger, new psychometric analysis of ASL-SUS (see section 4.6).

Psychometric analysis of ASL-NPS using data from studies 2018-US-ASL and 2018-US-ENG; presented in section 5.6

Stage 5: Distribute ASL-NPS materials and study data (5.5)

Fig. 1. Table of contents for this project.

after each (participants whom were part of the 2017 studies did not take part in the 2018 studies). These participant responses on a more diverse set of artifacts provide us with a stronger foundation for data analyses. Table 1 (on the next page) enumerates all of the user studies mentioned in this article; "Study Label" codenames appearing in the table correspond to the codenames in Figure 1. 
Table 1. List of Studies in This Project

\begin{tabular}{|c|c|c|l|}
\hline Study Label & Type of Study & \# Particip. & Details of Study \\
\hline $2017-B T-1$ & ASL-SUS Back-Translation Round 1 & 9 & Section 4.3 \\
\hline $2017-B T-2$ & ASL-SUS Back-Translation Round 2 & 10 & Section 4.3 .1 \\
\hline $2017-U S-A S L$ & Old User Study for ASL-SUS & 30 & {$[31]$} \\
\hline $2017-U S-E N G$ & Old User Study for English SUS & 10 & {$[31]$} \\
\hline $2018-B T-1$ & ASL-NPS Back-Translation Round 1 & 4 & Section 5.3 \\
\hline $2018-B T-2$ & ASL-NPS Back-Translation Round 2 & 12 & Section 5.3.1 \\
\hline $2018-U S-A S L$ & New User Study for ASL-SUS \& ASL-NPS & 30 & Section 4.4.1 \\
\hline $2018-U S-E N G$ & New User Study for English SUS and NPS & 12 & Section 4.4.3 \\
\hline
\end{tabular}

\section{CREATING THE ASL-SUS}

To begin our translation of SUS into ASL, we first selected which English version of the SUS instrument to use as the basis. Specifically, we chose to translate the "modified" SUS version as provided by $[4,11]$. As compared to the original version of SUS [10], the "modified" version slightly changed some wording that had been confusing for participants, e.g., "cumbersome" in Q8 was changed to "awkward," and all of the questions were updated to use "product" instead of "system" to reflect the broad usage of SUS. In addition to preferring this updated wording, we chose to use the "modified" version as a basis for our translation so our team could compare the validity of ASL-SUS to prior published research utilizing the modified version of SUS such as References [4, 5, 11, 64], and the many translation efforts documented in Section 2.3.

\subsection{Translation into ASL, Initial Revisions (Stage 1)}

During the first stage, a translation team was assembled, consisting of experts with skills in HCI and fluency in ASL and English:

- The first member was a doctoral student in computing, with several years of experience in the computing industry, who had completed four semester-long courses in HCI and was conducting research in HCI. This student was a fluent, native signer of ASL, born Deaf into a Deaf family of ASL-signers, and a graduate of a primary/middle/secondary school for the Deaf and from a university with instruction in ASL.

- The second member was a master's degree student in an HCI graduate program who had completed four semester-long courses in HCI, with experience conducting hundreds of hours of user testing, especially in-person studies with people who are $\mathrm{DHH}$. This student was a fluent, native ASL signer who was born Deaf and used ASL since birth, and a graduate of a primary/middle/secondary school for the Deaf and from a university with instruction in ASL and English.

- The third member was a faculty member with a $\mathrm{PhD}$ in computing who publishes research in HCI and accessibility, and who had experience in corpus-based ASL computational linguistics research. This faculty member was a fluent signer who learned ASL as an adult, having completed 10 university courses in ASL, including 2 summer ASL immersion programs, and regularly uses ASL with DHH lab members.

The members of the team fell into specific roles: The faculty member suggested translation options and particular ASL linguistic structures (e.g., rhetorical questions, use of reference points in the signing space, etc.) to convey the original SUS question meaning. The doctoral student had final authority on the fluency of the ASL translations, given this student's "Deaf-of-Deaf" background 
and native signing skills. The masters student identified terminology about technology likely understood by DHH participants in usability studies and when particular ASL options proposed by the doctoral student were so idiomatic as to be difficult to understand for a $\mathrm{DHH}$ participant who may possess non-native ASL fluency.

To begin the process, the team discussed how the ten SUS question items could be translated to ASL, and found it useful to use a mobile phone video camera to record themselves producing alternatives and saving brief video clips. Multiple revisions were made for each SUS item, through this process, until a consensus set of ASL items were produced. The team avoided using an overly word-for-word transliteration of the original English items, to avoid producing non-fluent Englishlike signing videos. Instead, the team strove to preserve the overall meaning of each item, while using ASL structure. These casually recorded videos were transcribed to produce an ASL script representing each item (see Appendix B for more details on our syntax for ASL scripts).

4.1.1 Adding Instructions for SUS. The translation team also created draft concepts for how the SUS instructions (presented at the beginning of the questionnaire) could be conveyed in ASL. In translating the instructions to ASL, additional explanatory content had to be added. For instance, the instructions had to explain that participants would need to watch videos of ASL and then circle items on paper, and the disagree/agree scale had to be explained in ASL (since the English questions would not appear on the paper). Further, the original SUS instructions mentioned the user's "reactions to the website today." Researchers generally change the word "website" to "device" or "software" as needed. To avoid producing multiple versions of our ASL videos, we needed the instructions to explain that the questions referred to the website, software, or device that the person had just used.

\subsection{Recording of High Quality ASL Videos (Stage 2)}

In stage two, high-definition videos were recorded of a native ASL signer (shown in Figure 2) performing each of the ASL-SUS items, in a video recording studio with professional-quality overhead lighting.

The ASL signer wore a solid-color black shirt, which contrasted with his skin color, and a plain blue background (contrasting with both the skin and shirt color) was behind the signer. The master's degree student on the translation team acted as a coach, behind the camera, with a copy of the script, observing the ASL produced by the native signer being recorded to ensure that the performance matched the intended ASL translations.

\subsection{Back-Translation, Final Revisions (Stage 3)}

In this third stage, we conducted a back-translation experiment to determine whether the translation preserved the meaning of the original. Two rounds of studies were conducted during this stage, as summarized in Table 2.

In the first back-translation study for ASL-SUS (2017-BT-1), we recruited nine participants (five female, four male) for this study, who were advanced students (third- or fourth-year) in a bachelor's degree program in ASL interpreting at Rochester Institute of Technology. Our rationale for recruiting interpreting students is that they regularly complete assignments in which they view and critique videos of ASL signing, and they have skills in discussing translation alternatives. The age of participants ranged from 20 to 26 (median $=22$ ). Three reported having deaf family members, including one participant who self-described as a Child of Deaf Adults (CODA). Aside from the CODA, who had used ASL since birth, the remaining participants had been using ASL for 3 to 8 years $($ mean $=5.25)$. Participants were paid $\$ 40$ for participating in this one-hour study, which was approved by the university IRB. 


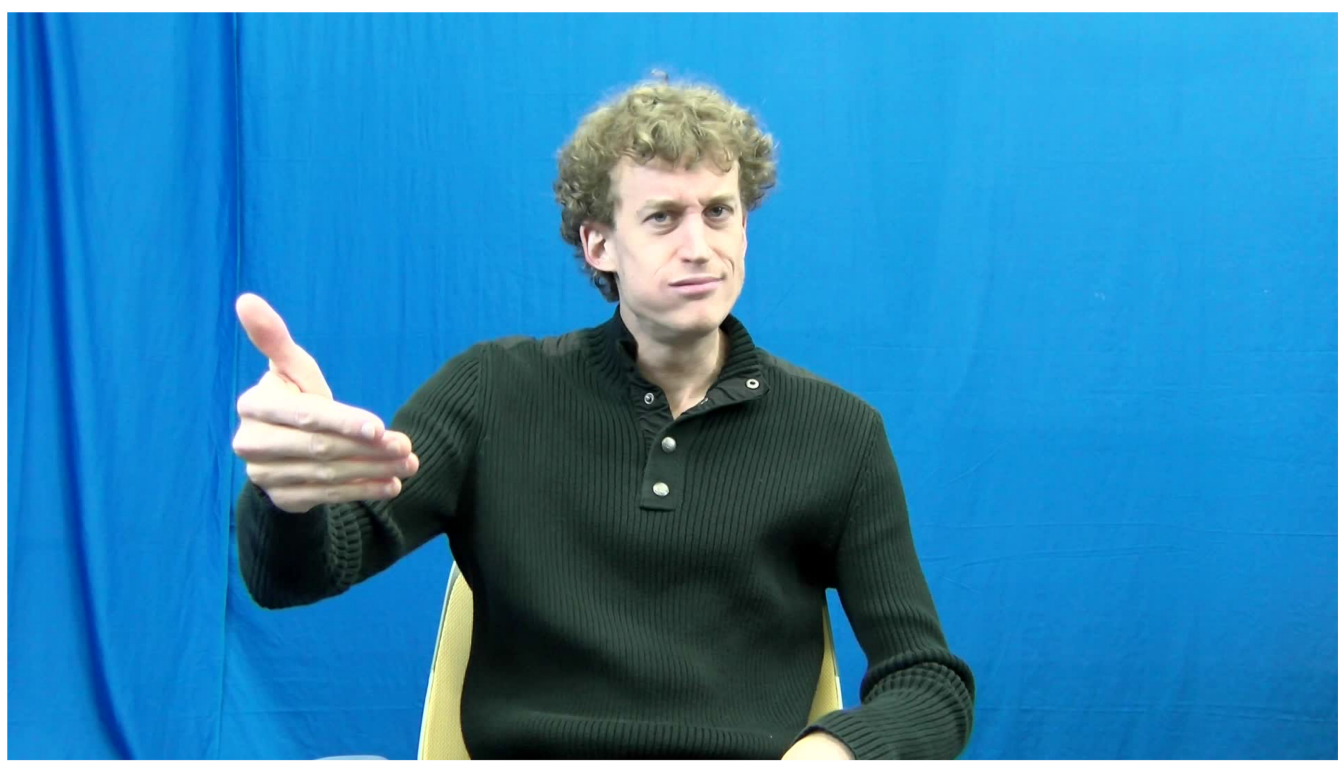

(a)

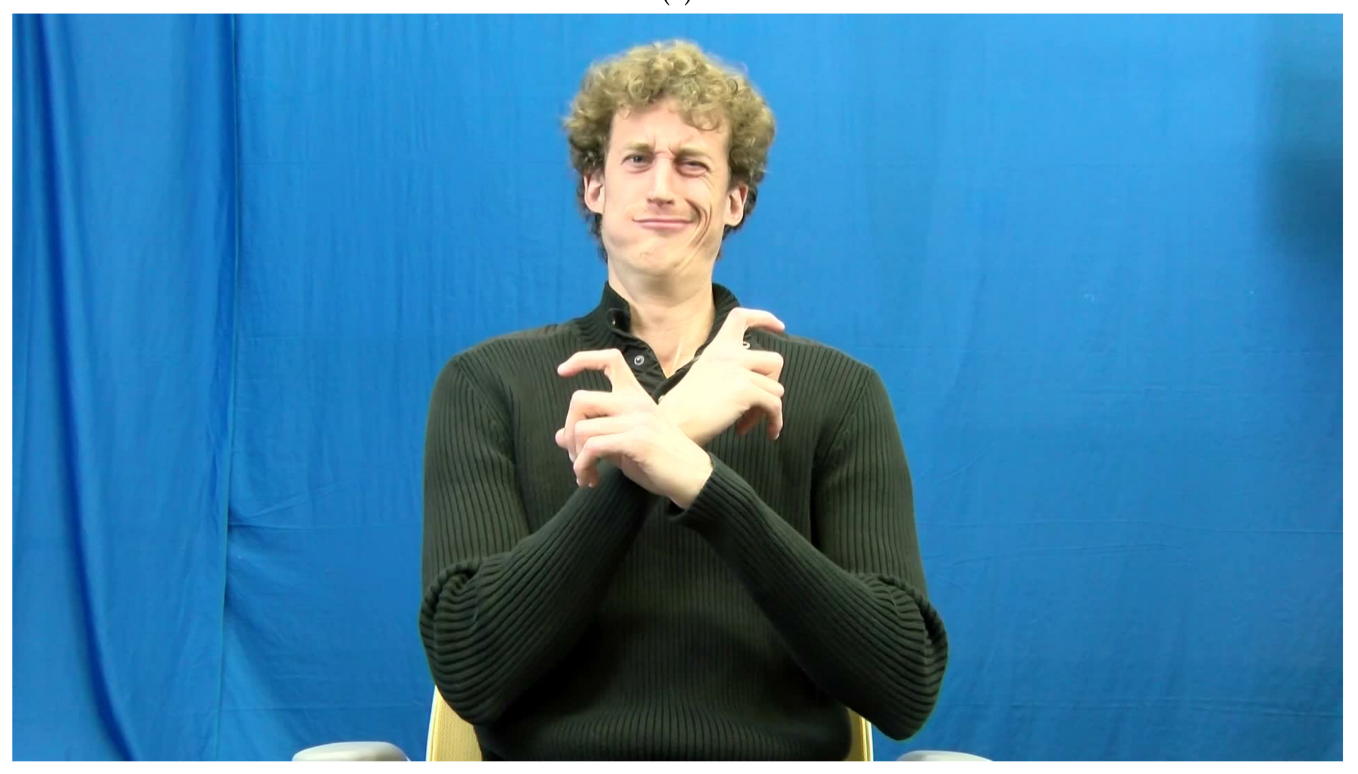

(b)

Fig. 2. Example screenshots from videos of a native ASL signer performing some of the ASL-SUS items: (a) excerpt from ASL-SUS for "I think that I would like to use this frequently," (Q1) showing ASL sign FREQUENT (at 7.55s), with cheeks-puffed-air-released-on-side facial expression to increase the degree of magnitude of the concept "frequently"; (b) excerpt from ASL-SUS for "I found the system unnecessarily complex," (Q2) showing ASL sign COMPLEX (at 8.2s), with a negative-grimace facial expression to indicate bemused irritation. 
Table 2. List of Back-Translation Studies for ASL-SUS (subset of items listed in Table 1)

\begin{tabular}{|c|c|c|l|}
\hline Study Label & Type of Study & \# Particip. & Activity \\
\hline 2017-BT-1 & ASL-SUS Back-Translation Round 1 & 9 & $\begin{array}{l}\text { Back-translated ASL-SUS } \\
\text { to English, shared critique } \\
\text { with researcher }\end{array}$ \\
\hline 2017-BT-2 & ASL-SUS Back-Translation Round 2 & 10 & $\begin{array}{l}\text { Back-translated ASL-SUS } \\
\text { to English, shared critique } \\
\text { with researcher }\end{array}$ \\
\hline
\end{tabular}

The participants were presented with each ASL-SUS video, and they were asked to write an English translation for each. Afterward, participants were shown the original English SUS, and they were asked to compare their translation to the original and to provide written feedback about any cases in which the meaning diverged. Participants were asked to offer suggestions about how the ASL video translation could be revised so that it would have been more successful at conveying the meaning of the original English item.

The translation team (Section 4.1) analyzed the back-translations and feedback comments from this study to identify any ASL videos for which the back-translations diverged from the meaning of the original English item or for which the participants had indicated suggestions of how the ASL video could have been improved. The translation team identified 2 items that needed to undergo major revisions based on the feedback, and 4 items that needed some small changes. Some examples of revisions made to our original ASL videos based on this feedback include:

- Our original translation of SUS question 5 "I found the various functions in the product were well integrated" into ASL was as follows: I LOOK THIS down $_{\text {, DO-DO }}$ repetitive-circular-sweeping cheek-puff MAINSTREAM-INTEGRATE \} check-puff-side-release\{ SMOOTH \}. (Appendix B describes our notation used for ASL transcription.) In the feedback comments, most participants indicated that they had not understood the signer's use of a repeated DO-DO sign performed in a circular sweeping arc motion to convey that the product does lots of things. For instance, one of the back-translations was "I can see all the pieces coming together." We decided to make use of the ASL sign ACTIVITY to more overtly refer to the many functions of the system. ${ }^{3}$ The revised ASL version of this item was: ILOOK THIS $_{\text {down }}$, MANY ACTIVITY (signer sets up a list buoy, ${ }^{4}$ with rhythmic nodding when pointing to each item) I CAN USE TOGETHER FINE, WAVE-WOW.

- The initial translation of SUS question 8 "I found the product very awkward to use" into ASL

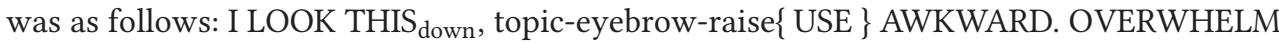
(shrug). Some participants indicated that the signer's performance of AWKWARD in the video was somewhat fast and difficult to perceive. Furthermore, several indicated that the use of OVERWHELM suggested that the fault lay with human user (who was not able to understand the technology), rather than the technology having some flaw. For instance, one back-translation was "This is over my head." Therefore, in the revised version of this item,

\footnotetext{
${ }^{3}$ While some ASL signers use a specific sign for "function," the native ASL signers on the translation team felt that this was an English-influenced sign, using an " $F$ " handshape initialization, and it may not be widely understood by signers less familiar with computer jargon.

${ }^{4}$ In ASL, a "list buoy" refers to a linguistic construction in which the signer raises the non-dominant hand into the signing space with some number of fingers extended, to represent a list of items that is being communicated. The signer generally points to each finger when referring to or introducing each item in the list. Here, the list buoy is used in a non-specific sense, to indicate that there are a multitude of functions.
} 
the translation team decided to omit the sign OVERWHELM, and the sign AWKWARD was slowed down (with a "disgust-tense" facial expression added) to improve intelligibility.

4.3.1 Second Round of Back-Translation for ASL-SUS. We confirmed that our revisions had addressed the concerns raised in the back-translation study by evaluating the revised videos in a second back-translation study (2017-BT-2) with 10 new participants (eight female, two male; ages 20 to 23; mean $=9.4$ years of ASL usage; all in the third and fourth year in the ASL interpreting degree program). For example, with SUS question 5, our team looked at the responses from the participants to evaluate whether the addition of ACTIVITY produced better results and some backtranslations with their comments are provided below:

- P1: "I'm able to use all the different actions this product has." -Comment: "Various functions not actions."

- P3: "I can easily use this product for many different purposes." - Comment: "My very minimal familiarity with technical language made me not think of the words 'functions or integrated' so I went much more general in my interpretation."

- P9: "There are many activities available to me." -Comment: "Functions' = activities?"

Our team reviewed all of the back-translations for each question item, and based on the comments from participants in the second round (2017-BT-2) our team decided to not revise the ASLSUS items any further, given the positive results.

\subsection{Summative User Study Evaluation (Stage 4)}

During the fourth stage, we evaluated our revised ASL-SUS items, by conducting several studies in which DHH participants evaluated the usability of several artifacts, as summarized in Table 3. DHH participants who were ASL signers were recruited from the Rochester Institute of Technology and surrounding community for these studies, which were approved by our university IRB. Participants were paid $\$ 40$ for participating in each one-hour study. A fluent ASL signer conducted each experimental session in ASL, and the participant was handed a sheet of paper with the list of tasks (written in English-see Appendix F for a listing of the tasks per artifact). After completing the tasks, the participants were asked to view the usability questionnaire instruments for each study.

The artifacts and tasks that we presented to participants in the user studies are (more details in Appendix F) shown in Table 4.

4.4.1 Summative User Study with ASL-SUS (US-ASL). In studies (2017-US-ASL and 2018-USASL) in which ASL-SUS responses were collected, after spending time interacting with each artifact, participants viewed an introductory video with instructions, followed by the ten Likert-type items. (Appendix C contains transcripts of the ASL-SUS items and a URL where the videos can be downloaded or viewed.) Participants indicated their answer choice for each Likert item on a piece of paper that contained a list of numbers from 1 to 10 arranged vertically (corresponding to each SUS question), with a set of five checkboxes next to each. The leftmost checkbox was labeled "Strongly Disagree," and the rightmost, "Strongly Agree." No other English questions or instructions appeared on the paper.

4.4.2 Utilizing the Bangor et al. Adjective Scale ("Adj"). To evaluate the criterion validity of ASL-SUS, it was necessary for our participants in the studies listed in Table 3 to also respond to another pre-existing usability instrument. In Reference [5], researchers had wanted to identify English adjectives that corresponded to various SUS scores [5]; so, they asked 964 English-speaking 
Table 3. List of User Studies to Gather Participant Responses to Instruments (subset of items from Table 1)

\begin{tabular}{|c|c|c|c|}
\hline $\begin{array}{l}\text { Study Label \& } \\
\text { Type of Study }\end{array}$ & Instruments & Artifact & Participant Details \\
\hline $\begin{array}{l}\text { 2017-US-ASL: } \\
\text { Old User Study } \\
\text { for ASL-SUS }\end{array}$ & $\begin{array}{l}\text { ASL-SUS, } \\
\text { ASL-Adj }\end{array}$ & $\begin{array}{l}\text { University Website } \\
\text { (long list of tasks) }\end{array}$ & $\begin{array}{l}\text { Thirty participants, with ages ranging } \\
\text { from } 19 \text { to } 27 \text { (mean }=22.5 \text { ), consisting } \\
\text { of } 15 \text { females and } 15 \text { males. Of the } 30,25 \\
\text { self-identified as Deaf, and 5, as } \\
\text { Hard-of-Hearing. Twenty participants } \\
\text { had used ASL since infancy, } 6 \text { since age } \\
3 \text {, and the remainder by age } 5 \text {. Eleven } \\
\text { participants indicated that they had } \\
\text { grown up in households with ASL } \\
\text { signing parents. }\end{array}$ \\
\hline $\begin{array}{l}\text { 2017-US-ENG: } \\
\text { Old User Study } \\
\text { for English SUS }\end{array}$ & $\begin{array}{l}\text { English SUS, } \\
\text { English Adj }\end{array}$ & $\begin{array}{l}\text { University Website } \\
\text { (long list of tasks) }\end{array}$ & $\begin{array}{l}\text { Ten participants, with ages ranging } \\
\text { from } 20 \text { to } 27 \text { (mean }=22.4 \text { ), consisting } \\
\text { of } 4 \text { females and } 6 \text { males. Of the } 10,5 \\
\text { self-identified as Deaf, and } 5 \text {, as } \\
\text { Hard-of-Hearing. Three had used ASL } \\
\text { since infancy, } 2 \text { since age } 4,2 \text { since age } \\
7 \text {, and the remainder by age } 16 \text {. Five } \\
\text { participants indicated that they had } \\
\text { grown up in households with ASL } \\
\text { signing parents. }\end{array}$ \\
\hline $\begin{array}{l}\text { 2018-US-ASL: } \\
\text { New User Study } \\
\text { for ASL-SUS } \\
\text { and ASL-NPS }\end{array}$ & $\begin{array}{l}\text { ASL-SUS, } \\
\text { ASL-Adj, } \\
\text { ASL-NPS }\end{array}$ & $\begin{array}{l}\text { University Website, } \\
\text { Government } \\
\text { Website, Tablet } \\
\text { Real-Estate App, } \\
\text { Digital Camera }\end{array}$ & $\begin{array}{l}\text { Thirty participants, with ages ranging } \\
\text { from } 19 \text { to } 46 \text { (mean = 23.9), consisting } \\
\text { of } 16 \text { females and } 14 \text { males. Of the } 30,21 \\
\text { self-identified as Deaf, } 8 \text { as } \\
\text { Hard-of-Hearing, and one as both Deaf } \\
\text { and Hard-of-Hearing. Thirteen } \\
\text { participants had used ASL since } \\
\text { infancy, } 8 \text { learned ASL before age 10, } \\
\text { and the remainder by age } 18 \text {. Sixteen } \\
\text { participants indicated that they had } \\
\text { grown up in households with ASL } \\
\text { signing parents, with } 5 \text { participants } \\
\text { whose parents were DHH. }\end{array}$ \\
\hline $\begin{array}{l}\text { 2018-US-ENG: } \\
\text { New User Study } \\
\text { for English SUS } \\
\text { and NPS }\end{array}$ & $\begin{array}{l}\text { English SUS, } \\
\text { English Adj, } \\
\text { English NPS }\end{array}$ & $\begin{array}{l}\text { University Website, } \\
\text { Government } \\
\text { Website, Tablet } \\
\text { Real-Estate App, } \\
\text { Digital Camera }\end{array}$ & $\begin{array}{l}\text { Twelve participants, with ages ranging } \\
\text { from } 18 \text { to } 25 \text { (mean }=22 \text { ), consisting of } \\
3 \text { females and } 9 \text { males. Of the } 12,8 \\
\text { self-identified as Deaf, } 3 \text { as } \\
\text { Hard-of-Hearing, and one as } \\
\text { Hard-of-Hearing/Oral. Six learned ASL } \\
\text { before age } 5,3 \text { before age } 10 \text { and the } \\
\text { remainder by age } 18 \text {. Five participants } \\
\text { indicated that they had grown up in } \\
\text { households with ASL signing parents, } \\
\text { and one had parents whom were DHH. }\end{array}$ \\
\hline
\end{tabular}


Table 4. Artifacts and Tasks for the 2018-US-ASL and 2018-US-ENG User Studies

\begin{tabular}{|l|l|l|}
\hline Artifact Type & \multicolumn{1}{|c|}{ Artifact Details } & \multicolumn{1}{c|}{ Summary of Tasks } \\
\hline University Website & $\begin{array}{l}\text { Rochester Institute of } \\
\text { Technology: https://www.rit.edu }\end{array}$ & $\begin{array}{l}\text { Information-seeking: event } \\
\text { calendar, phone numbers, etc. }\end{array}$ \\
\hline Government Website & $\begin{array}{l}\text { Monroe County Government: } \\
\text { https://www.monroecounty.gov }\end{array}$ & $\begin{array}{l}\text { Information-seeking: } \\
\text { certifications, recycling fees, etc. }\end{array}$ \\
\hline Tablet App & $\begin{array}{l}\text { Trulia: https://play.google.com/ } \\
\text { store/apps/details?id=com.trulia. } \\
\text { android }\end{array}$ & $\begin{array}{l}\text { Information-Seeking: rentals that } \\
\text { allow dogs, monthly property } \\
\text { taxes, etc. }\end{array}$ \\
\hline Digital Camera & $\begin{array}{l}\text { LLION 2.7" HD Mini: } \\
\text { https://www.amazon.com/gp/ } \\
\text { product/B077XM7TXM }\end{array}$ & $\begin{array}{l}\text { Exploratory: taking pictures / } \\
\text { movies, changing scene settings, } \\
\text { etc. }\end{array}$ \\
\hline
\end{tabular}

participants to evaluate a variety of websites and software, across several studies. The participants responded to the original English version of the SUS questionnaire, and in addition, they responded to a novel seven-point "adjective scale" designed by the researchers. Participants were asked to select a word that indicated how user-friendly they believed the website or software to be. The scale consisted of adjectives, scored as ordinal values: Worst Imaginable (1), Awful (2), Poor (3), OK (4), Good (5), Excellent (6), Best Imaginable (7). We decided to ask our participants to respond to this adjective scale in our study too: since this instrument had previously been compared to the original English SUS, we could perform a similar analysis of our new ASL-SUS. Since our participants were fluent ASL signers, we provided an ASL video translation of the Adjective Scale user-friendliness question (ASL-Adj) and the answer choice levels (see Appendix D), but the paper answer-sheet presented the seven options using the original English adjectives.

4.4.3 Comparison Study with English SUS (US-ENG). While there are many published results and norms available for English-speaking users responding to original English SUS items, we wanted to gather a small set of response data from additional DHH participants evaluating the same artifacts as in the US-ASL studies. Section 4.6 discusses how this comparative data is used to evaluate the ASL-SUS. The procedure and tasks in those studies (2017-US-ENG and 2018-USENG) were identical to those in study US-ASL, with one difference: Instead of responding to the ASL-SUS (and the ASL-Adj), these participants responded to the original English items on a paper questionnaire with no ASL explanation of the questions.

4.4.4 Changes Made to Studies as We Collected Additional Data. The data from two of these studies (2017-US-ASL and 2017-US-ENG) had been collected and used as the basis for our evaluation of SUS in our prior conference paper [31] (in the paper, those studies were labeled \#1 and \#2, respectively). In both cases, participants responded to the usability instruments after interacting with a single artifact (a university website, as described in Table 4). We used a methodology similar to a prior study that had utilized SUS [12]: We asked participants to access the website and perform information-seeking tasks, without using a search engine.

Concerned that our initial dataset of participant responses to SUS was based on reactions to a single artifact, we subsequently conducted two additional studies (2018-US-ASL and 2018-USENG), with a goal of diversifying the set of artifacts that participants evaluated, and with an additional goal of gathering responses to our newly developed ASL-NPS instrument (details in Section 5). In these newer studies, participants responded to an entire battery of question items (ASL-SUS, ASL-Adj, and ASL-NPS), so that we could collect additional data to support our psychometric evaluation effort (refer to Figure 1 for more details on how we organized this project). 
In our initial studies presented in Reference [31], we found that participants needed 30min to complete the long sequence of tasks they were given for the university website, prior to their responding to the usability instruments. Mindful of the finding of [63] that reducing the timeduration of users interacting with an artifact had a very small impact on SUS scores, and hoping to avoid user fatigue, we decided to keep the sequence of tasks for any single artifact to under $5 \mathrm{~min}$, with $10 \mathrm{~min}$ of time after each for the participant to respond to the usability instruments. In this way, we sought to enable a participant to evaluate three or four artifacts during a one-hour session. After a small pilot test, we found that evaluating four artifacts was doable in a single $60 \mathrm{~min}$ session (with only a few tasks for each artifact rather than a long list as in the prior work). Details of what we asked participants to do with each artifact appear in Appendix F.

To diversify the artifacts that participants evaluated, we added a tablet app and a digital camera, with a set of tasks for each. Researchers in the aforementioned English-to-Polish SUS study [9] recommended avoiding the use of a "popular" webpage, e.g., Facebook, and to include systems with varying user-friendliness levels. One reviewer of our original conference paper suggested that we ask participants to evaluate a government webpage with a lower level of expected literacy, we picked our local county's webpage. Finally, to maintain some consistency with the original pair of studies, we retained the university website, but reduced the number of tasks participants were asked to perform. We used a Latin-squares style to rotate the order of artifacts presented to participants.

The results and analysis presented in Section 4.6 differ from those that had been presented in our original conference paper [31]. Specifically, our new analyses are based on the entire set of participant response data, from all four studies listed in Table 3. Thus, for analysis of ASLSUS results, we have combined the data from studies 2017-US-ASL and 2018-US-ASL to produce a single dataset with data from 60 participants. Similarly, for comparative analysis with English SUS results, we have combined the data from studies 2017-US-ENG and 2018-US-ENG to produce an amalgamated dataset (22 participants in total). The user studies' datasets could be safely combined without skewing our analyses as our team is not interested in the raw values participants provided for each artifact; we are evaluating the instrument (ASL-SUS), rather than the artifact itself.

\subsection{Dissemination of ASL-SUS (Stage 5)}

In the final stage of our study, we have disseminated our new ASL-SUS (and ASL-Adj), to share this resource with the research community. Appendix C provides a transcript of each SUS question item and Appendix D provides a transcript of ASL-Adj. The final version of the ASL videos and PDFs of the paper answer sheets from the older studies are available at http://latlab.ist.rit.edu/ taccess2019asl. Furthermore, the entire collection of videos, answer sheets, and raw numerical response data (amalgamated) is also available in the ACM Digital Library as an online appendix: Appendix H.

\subsection{ASL-SUS Results}

This section analyzes the results from our evaluation of ASL-SUS, with a focus on addressing the research questions in Section 3. We want to remind the reader that the results in this section are previously unpublished, as they are based on a combination of data from user studies 2017-USASL, 2017-US-ENG, 2018-US-ASL, and 2018-US-ENG. Please refer to Figure 1 in the Introduction to see how the four user-studies have been used in this project. We will refer to the combined dataset as "US-ASL" and "US-ENG" for the remainder of this section.

Our first research question (RQ1) was whether we had preserved the meaning of the original English SUS during the translation process used to produce ASL-SUS. This issue was primarily examined in a formative manner, during the translation and back-translation evaluation process. 
To ensure that meaning was preserved during translation, we used several commonly accepted methodologies: a team of experts produced the translation, multiple rounds of revision occurred, a back-translation evaluation was conducted prior to the final revision, and native ASL signers participated in the translation process. Informally, we note that the mean scores for the original English SUS (52.11) and the ASL-SUS (56.65) were quite similar ${ }^{5}$; this is a reasonable result, since DHH participants evaluated identical artifacts and performed identical tasks in both studies. A Mann-Whitney U-test (also known as Wilcoxon rank-sum test with continuity correction) was performed to compare the means, and the difference was not significant $(U=4856, p=0.1938)$.

Although not a formal research question identified in this study, we were also interested in the overall usability of the ASL-SUS, i.e., whether DHH participants found any of the videos confusing or difficult to understand. During the summative study (Section 4.4.1), we invited participants to indicate if they were unsure of the meaning of any ASL-SUS videos or had difficulty in responding to any questions. At the end of the study, participants wrote feedback comments about their experience participating in the study. None of the 60 participants indicated that they experienced difficulty in understanding or responding to the ASL-SUS questions.

In regard to RQ2, Sections 4.6.1-4.6.3 analyze the results from studies US-ASL and US-ENG to examine three key psychometric properties: internal reliability, criterion validity, and construct validity.

4.6.1 Internal Reliability. To evaluate the internal reliability of ASL-SUS, i.e., whether all items contribute to the overall score, we calculated ${ }^{6}$ Cronbach's Alpha [16]. Since even-numbered items on SUS are reverse-scored (they have negative polarity), we inverted those items. For responses from 60 participants in the study US-ASL, the Alpha for ASL-SUS was 0.898 with $95 \%$ CI [0.88, 0.92]. Generally, Alpha scores of 0.9 and above are considered excellent [37].

To assess the internal reliability of our new ASL-SUS, we compare this Alpha value above with two other Alpha values:

- In a prior study examining the internal reliability of the original English SUS, researchers reported Alpha scores of 0.911, based on responses from 2,324 hearing participants [4].

- In our US-ENG studies, 22 DHH participants responded to the original English SUS, while evaluating identical artifacts as in study US-ASL; we calculated an Alpha value of 0.895 with $95 \%$ CI $[0.85,0.94]$.

Given the Alpha value of 0.898 for ASL-SUS in study US-ASL (and the relatively similar Alpha values for English SUS in study US-ENG, also with DHH participants), we conclude there is strong evidence for the internal reliability of the ASL-SUS. We have noticed recent publications [13, 21, $58,74]$ that advocate for researchers to discontinue using Cronbach's Alpha in favor of newer statistical methods. We have still published Alpha values in this article, to enable comparison with prior published values for SUS, but Appendix G includes results from several alternative methods for calculating internal reliability.

4.6.2 Criterion Validity. One method of evaluating criterion validity is to determine how convergent an instrument is with other instruments that measure the same concept. Section 4.4 described how our participants, in addition to responding to ASL-SUS, also responded to ASL-Adj. To assess whether the scores from each participant on the ASL-SUS were correlated to the scores from that participant on the ASL-Adj, we calculated the Pearson's coefficient $r=0.759, p<0.001$.

${ }^{5}$ Per-artifact means: (RIT: 60.45 for SUS, 62.83 for ASL-SUS), (Monroe: 30.21 for SUS, 42.75 for ASL-SUS), (Trulia: 62.08 for SUS, 63.75 for ASL-SUS), and (Camera: 48.75 for SUS, 51.08 for ASL-SUS).

${ }^{6} \mathrm{We}$ used the R programming language [52] and the psych package [56] to compute all of the results in this article. 
This result indicated a significant correlation ${ }^{7}$ between participants' responses to these instruments.

To assess the criterion validity of ASL-SUS, we compare this correlation above with two other correlations:

- Previously Published Correlation between English SUS and English Adj: In Reference [5], the authors calculated the correlation between their participants' English SUS scores and their English Adj scores: Pearson's $r=0.822, p<0.01$. We performed a Fisher's $r$-to-z transformation to determine whether this ASL-SUS-to-ASL-Adj correlation from study US-ASL was significantly different than the English-SUS-to-English-Adj correlation reported in Reference [5]; no significant difference was observed ( $p=0.0561, z=1.91$ ).

- Study US-ENG Correlation between English SUS and English Adj: The participants who provided the response data in Reference [5] consisted almost entirely of hearing participants. To compare our study US-ASL correlation above with data from a more similar group of users, we can examine the data from study US-ENG, in which $22 \mathrm{DHH}$ participants responded to the original English SUS and to the English Adjective scale. We calculated the correlation between SUS and Adj for these users: Pearson's $r=0.822, p<0.001$. To compare our study US-ASL results and study US-ENG results, a Fisher's r-to-z transformation was performed to determine whether our study US-ASL ASL-SUS-to-ASL-Adj correlation was significantly different than the study US-ENG English-SUS-to-English-Adj correlation; no significant difference was observed ( $p=0.2846, z=1.07$ ).

Thus, we conclude that there is strong evidence that the ASL-SUS has comparable criterion validity to the original English SUS, using the English Adjective scale of Reference [5] as our criterion.

4.6.3 Construct Validity. A prior study [5] concluded that SUS had a single factor structure (based on an examination of the results of a single-factor analysis), yet researchers in Reference [42] discuss how specifically investigating multi-factor solutions led them to conclude that SUS actually consists of two factors: "Learnable" (based on the responses to items 4 and 10) and "Usable" (based on the responses to the remaining items). Presented below is our evaluation of the construct validity of ASL-SUS, by determining whether this factor structure had been preserved during the process of translating the items to ASL.

Before discussing our factor analyses, it is important to note that the methodology in Reference [42] was slightly unorthodox: the authors did not use a rigorous statistical method to conclude that the SUS had a two-factor structure; they simply ran factor analyses with varying $\mathrm{N}$ and observing whether the results from their data converged with an alternative dataset from Bangor et al. [4]. Following best practices recommended in References [15, 61, 83], we utilized several methods to determine the number of factors in ASL-SUS before proceeding with the exploratory factor analysis. Using "Parallel Analysis" with minres for the factor method, we obtained 2 factors for ASL-SUS using both Principal Component Analysis (PCA) and Factor Analysis (FA) methods. Similarly, utilizing the Very Simple Structure (VSS) method [57] in conjunction with the Velicer minimum average partial (MAP) [84] resulted in a two-factor structure for ASL-SUS.

With the two-factor structure of ASL-SUS confirmed, it was possible to run Exploratory Factor Analysis (EFA) and Confirmatory Factor Analysis (CFA) methods on our dataset. Since the factor structure of SUS was already published in Reference [42], we executed ${ }^{8}$ a CFA fit on our dataset

\footnotetext{
${ }^{7}$ We also calculated Spearman's rho: 0.758 with $p<0.001$ for ASL-SUS to ASL-Adj.

${ }^{8}$ The CFA was performed using the lavaan package [59] for R. The CFA equation was: "Usable" = Q1 + Q2 + Q3 + Q5 + $\mathrm{Q} 6+\mathrm{Q} 7+\mathrm{Q} 8+\mathrm{Q} 9$, "Learnable" $=\mathrm{Q} 4+\mathrm{Q} 10$.
} 
Table 5. Factor Loadings for ASL-SUS and Previously Published Loadings for SUS

\begin{tabular}{|l|c|c|c|c|c|c|}
\hline Item & $\begin{array}{c}\text { Bangor } \\
\text { Usable }\end{array}$ & $\begin{array}{c}\text { Bangor } \\
\text { Learnable }\end{array}$ & $\begin{array}{c}\text { Lewis } \\
\text { Usable }\end{array}$ & $\begin{array}{c}\text { Lewis } \\
\text { Learnable }\end{array}$ & $\begin{array}{c}\text { ASL-SUS } \\
\text { Factor1 }\end{array}$ & $\begin{array}{c}\text { ASL-SUS } \\
\text { Factor2 }\end{array}$ \\
\hline Q1 & $\mathbf{0 . 7 0}$ & 0.22 & $\mathbf{0 . 7 1}$ & 0.21 & $\mathbf{0 . 6 7}$ & 0.19 \\
\hline Q2 & $\mathbf{0 . 5 9}$ & 0.38 & $\mathbf{0 . 6 2}$ & 0.46 & 0.48 & $\mathbf{0 . 7 0}$ \\
\hline Q3 & $\mathbf{0 . 7 1}$ & 0.45 & $\mathbf{0 . 6 9}$ & 0.43 & $\mathbf{0 . 6 9}$ & 0.50 \\
\hline Q4 & 0.27 & $\mathbf{0 . 6 9}$ & 0.28 & $\mathbf{0 . 5 8}$ & 0.24 & $\mathbf{0 . 7 5}$ \\
\hline Q5 & $\mathbf{0 . 7 1}$ & 0.23 & $\mathbf{0 . 6 0}$ & 0.26 & $\mathbf{0 . 7 3}$ & 0.13 \\
\hline Q6 & $\mathbf{0 . 5 8}$ & 0.39 & $\mathbf{0 . 5 8}$ & 0.39 & 0.12 & $\mathbf{0 . 5 0}$ \\
\hline Q7 & $\mathbf{0 . 6 4}$ & 0.36 & $\mathbf{0 . 6 2}$ & 0.46 & $\mathbf{0 . 6 1}$ & 0.29 \\
\hline Q8 & $\mathbf{0 . 6 0}$ & 0.41 & $\mathbf{0 . 7 7}$ & 0.38 & 0.38 & $\mathbf{0 . 7 6}$ \\
\hline Q9 & $\mathbf{0 . 6 0}$ & 0.52 & $\mathbf{0 . 6 4}$ & 0.47 & $\mathbf{0 . 6 9}$ & 0.46 \\
\hline Q10 & 0.31 & $\mathbf{0 . 6 9}$ & 0.32 & $\mathbf{0 . 7 9}$ & 0.21 & $\mathbf{0 . 6 8}$ \\
\hline
\end{tabular}

first to determine the goodness-of-fit. Following advice in Reference [34], the CFA had a good (but not great) fit when considering several metrics: Comparative Fit Index $(\mathrm{CFI})=0.881$, TuckerLewis Index $(\mathrm{TLI})=0.842$, Root-mean-square Error of Approximation $(\mathrm{RMSEA})=0.137$ with $95 \%$ CI $[0.112,0.162] p<0.001$, and the Standardized Root-mean-square Residual (SRMR) $=0.074$. To probe deeper into the factor structure and understand the differences between ASL-SUS and the published data for SUS, we then performed an EFA on our data: shown in Table 5 are the factor loadings from our dataset in conjunction with the loadings discovered in Reference [42].

Table 5 indicates that the factor loadings for ASL-SUS were similar to the original English SUS (Q4 and Q10 were loaded on the same factor), but the loading for some items was swapped: Q2, Q6, and Q8. Furthermore, the proportion of variance per factor in ASL-SUS was different than the prior data: $28 \%$ for Factor1, 29\% for Factor2 (Bangor: 35\% for Usable, 21\% for Learnable, Lewis: 36\% for Usable, $22 \%$ for Learnable) but the total variance was similar: 57\%. Following advice in References $[18,46]$, the Tucker index of factor congruence (Tucker's Phi) was used with Procrustean rotation to compare the loadings discovered for ASL-SUS with the loadings from Bangor et al. and Lewis and Sauro in Reference [42]. The Phi for Factor1 compared to Bangor is 0.97, and Lewis is 0.97. The Phi for Factor2 compared to Bangor is 0.94, and Lewis is 0.95. Based on the guidelines from Reference [46], Factor1 in ASL-SUS is considered equal to Usable, and Factor2 is considered "fairly similar" to Learnable.

Finally, with the factor structure confirmed for ASL-SUS to be very similar to SUS, we replicated the analyses done by Lewis and Sauro [42] on the Usable/Learnable scales: correlation and reliability. Using the same factor structure for Usable/Learnable on our data, the correlation is 0.69 (similar to the prior finding of 0.664), and re-using the structure found in our EFA, the correlation for Factor $1 /$ Factor2 is 0.65 , which was strikingly similar to the prior correlation. Last, we also calculated the reliability of the scales: the Alpha ${ }^{9}$ for Usable and Learnable on our data were, respectively, 0.881 and 0.699 (very similar to the published data: 0.91 and 0.70 ). Using the scales from the EFA, the Alpha ${ }^{10}$ for Factor1 and Factor2 were, respectively, 0.853 and 0.861.

After considering the strength of the evidence, we concluded that the construct validity of ASLSUS were comparable to SUS, using the extensive analysis of the factor structure of ASL-SUS as our criterion.

\footnotetext{
${ }^{9}$ We also calculated McDonald's Omega (Total): 0.887 for Usable, 0.699 for Learnable.

${ }^{10}$ We also calculated McDonald's Omega (Total): 0.859 for Factor1, 0.863 for Factor2.
} 


\section{CREATING THE ASL-NPS}

As discussed in the introduction (Section 1.1), our team wanted to repeat the English-to-ASL process with an alternative instrument, as to collect additional data and provide the community with more ASL translations of popular survey instruments. From the list of standardized instruments shown in Section 2.2, we picked the NPS as it was popular (e.g., used by large retail, hospitality, telecommunications, and other companies to evaluate customer-service experiences or customer satisfaction), and it is freely available without licensing restrictions. In addition, it has been used in conjunction with SUS in several studies, and correlation values between the NPS and SUS instruments was shown in Reference [64].

We found two papers that described translation efforts for NPS:

- An English-to-Polish team [9] used the NPS in their battery of questions, but they did not describe in detail how they translated NPS (as they simply included a commercial version from http://www.marketingowiec.pl) and used a slightly altered version of NPS: "Unlike the original, ten position scale range from 1 to 10 was used to be consistent with other questions used in the survey and to eliminate the middle answer." [9, p. 147]

- The team from Seth et al. [69] wanted to find out why NPS scores typically were lower in Asian countries when compared to other areas in the world, and pinpointed cultural differences as the major factor (the conceptual difference between evaluating the company's performance and recommending the company to a friend) [69]. Our team agreed that it was critical to consider cultural differences (as discussed in Section 2.4), and that we would need to carefully consider the difference between "company/product" and the "recommendation" aspect of the NPS for the Deaf community.

The following subsections describe our translation process (using the same methodology utilized for the ASL-SUS effort).

\subsection{Translation into ASL, Initial Revisions (Stage 1)}

For our initial translation stage, we needed to re-constitute the translation team: Since our initial conference paper, one member of our team graduated (the masters student-see Section 4.1), so we recruited another Deaf masters student in HCI to join our team with a similar educational and linguistic background to the student who had graduated.

The original English text of the NPS is as follows: "How likely is it that you would recommend our company to a friend or colleague?" with the answer scale ranging from 0 (Not at all likely) to 5 (neutral) to 10 (Extremely likely) [53]. Although some companies who analyze the results of NPS would be unhappy if their scores were below 7 (and consider this a poor result) [54, 55], this conceptualization of the scale is not revealed to the end-user who is responding to the instrument. Instead, when the NPS scale is presented to participants, the center-point on the scale (5) is presented as the "neutral" option [53], and we follow this convention in how we translate and present the scale here. Similar to the reasoning behind creating the ASL-SUS introduction video (Section 4.1.1), we generalized the word "company" to USE THIS ${ }_{\text {down }}$, so that we would not need to create several versions of the NPS for each artifact. Similar to the process used for ASL-SUS, we recorded several draft videos of the translation and shared it amongst the team members for discussion before arriving at the final draft version.

Shown below is the draft version we produced for the back-translation studies (see Section 5.3), we are providing it here so the reader can understand the changes the back-translation group recommended. A final version of the ASL-NPS appears in Appendix E. (Appendix B describes our notation used for ASL transcription.) 
- Draft of ASL-NPS:

topic-eyebrow-raise\{ YOU $\}$ USE THIS $_{\text {down }}$ FINISH.

conditional\{ NOW \} DREAM-BUBBLE-IMAGINE role-shift-gaze-right\{ MEET FRIEND \}. rhetorical\{ QUESTION WHAT $\}$ THIS $_{\text {down }}$

yes-no-question\{ WILL YOU role-shift-gaze-right INFORM \} (pauses with gaze at camera)\} topic-eyebrow-raise\{ ANSWER ZERO \} HAND-SWEEP topic-eyebrow-raise\{ TEN \}.

topic-eyebrow-raise $\{$ ZERO $\}$ head-shake-no\{ REFUSE $\}$

role-shift-gaze-right $\{$ INFORM $\}$ THIS $_{\text {down }}$ DISCARD.

HAND-SWEEP topic-eyebrow-raise\{ FIVE $\}$ MAYBE SO-SO.

HAND-SWEEP topic-eyebrow-raise\{ TEN $\}$ head-nod-yes $\{$ MUST $\}$

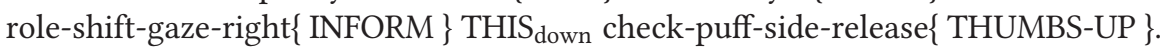

Readers familiar with ASL may have noted that the ASL translation above does not make use of the ASL sign SUGGEST (sometimes this sign is alternatively referred to as "RECOMMEND"), which consists of the open hands in a palms-up orientation, move forward from the torso and lifting upward.

There are some ASL signers who may even use the English word "RECOMMEND" as an alternate gloss (informal one-word translation, used when referring to ASL signs when discussing them in English) for the ASL sign SUGGEST, and moreover, some individuals who use an English-like manual communication systems may even perform the sign SUGGEST with an "R" handshape on both hands, to specifically transliterate the English word "recommend."

Yet, despite this context, our translation team intentionally chose to avoid using this sign, at the behest of the native ASL signer on our translation team who felt that the ASL sign SUGGEST is used in a different sense than the English word "recommend." While the ASL sign SUGGEST can be used to convey the concept of recommending an idea or a plan-of-action to someone, the use of SUGGEST in the sense of "expressing a positive attitude about something to urge someone to engage with it" is an English-like use of the word. Given the native ASL signer's concerns about this English-like usage, our ASL translation avoids this sign. Notably, participants in the second back-translation study (2018-BT-2) described in Section 5.3.1 were satisfied with our ASL version that avoided this particular sign.

\subsection{Recording of High Quality ASL Videos (Stage 2)}

For stage two, we utilized the same recording equipment as described in Section 4.2 for ASL-SUS and sample screenshots can be seen in Figure 3.

\subsection{Back-Translation (Stage 3)}

Once again, we conducted two rounds of back-translation studies (2018-BT-1 and 2018-BT-2)-this time, to evaluate the accuracy of our translation of NPS to ASL. These two studies are summarized briefly in Table 6 .

After producing the draft version of NPS, our team began the back-translation studies. When conducting these studies for ASL-SUS, we found that it was difficult to unify the various suggestions and comments from the interpreter participants in the first round of the study. For this reason, we tried a slightly different methodology for ASL-NPS: Rather than conducting two rounds of back-translation studies with individual participant appointments, we decided to conduct the first round (2018-BT-1) as a focus group, to enable more interactive discussion and explanation from participants. For this first back-translation round (2018-BT-1), we invited two professional, certified ASL interpreters with many years of experience working at our university, and two Deaf students whom were working at the lab: one undergraduate student in $\mathrm{HCI}$, and one $\mathrm{PhD}$ student 


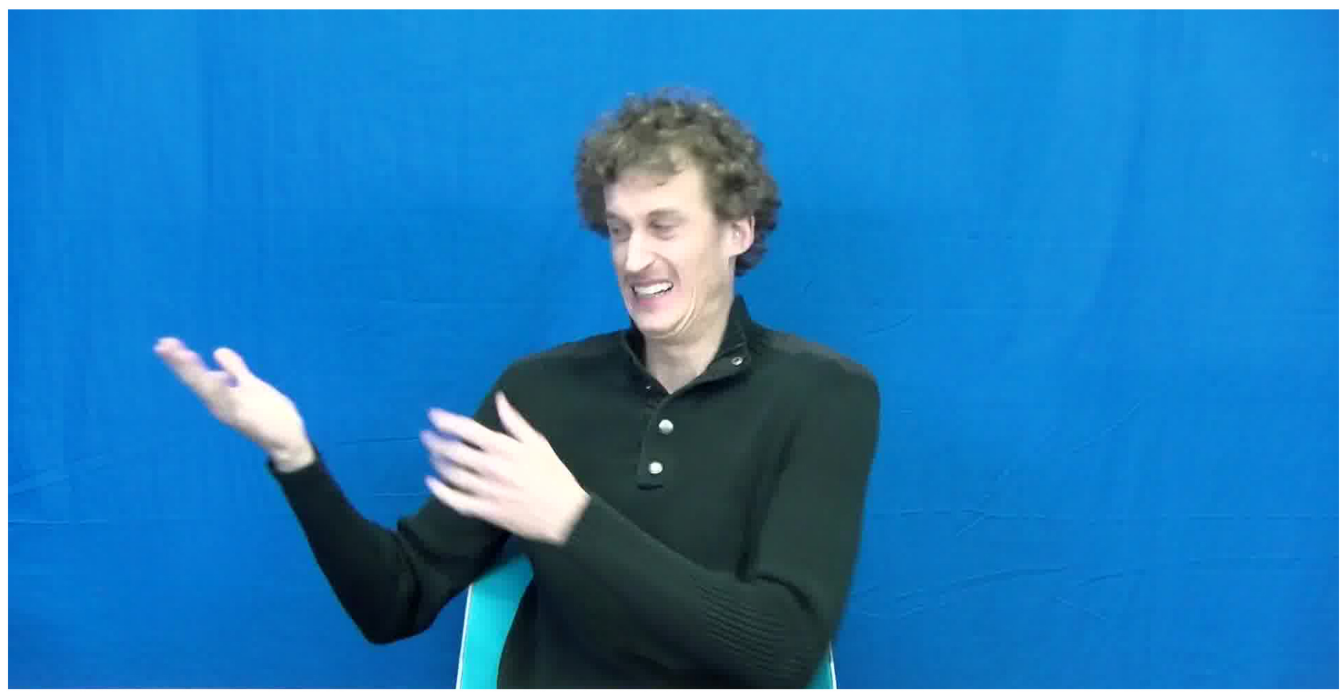

(a)

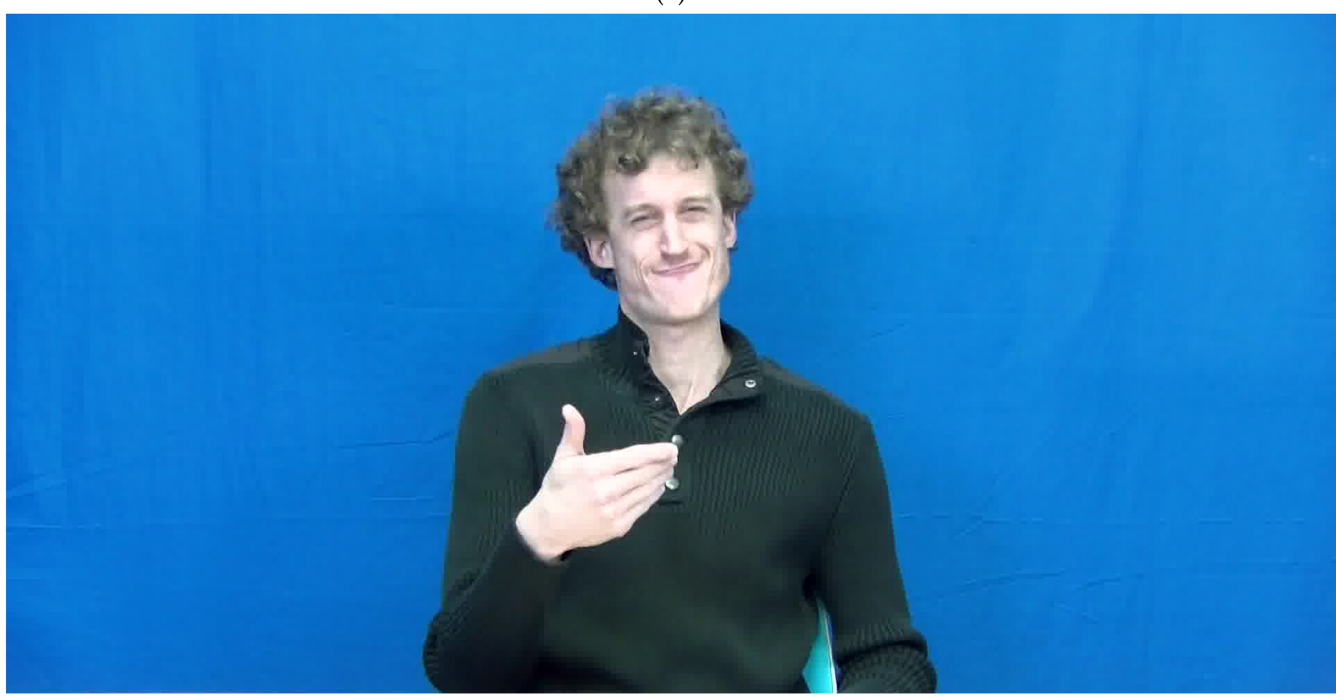

(b)

Fig. 3. Example screenshots from videos of a native ASL signer performing the ASL-NPS: (a) Excerpt from ASL-NPS, showing ASL sign COME-COME (at 14.5s), in which the signer is making a beckoning motion with his hand, with an teeth-baring facial expression (indicating intensity or urgency), while engaged in a role shift to his right side ${ }^{11}$; (b) Excerpt from ASL-NPS showing ASL sign DISCARD (at 28.5s), in which the signer is making a sweeping motion with his hand (as if brushing something aside), while looking at the camera and making a crooked grimace, indicating dissatisfaction.

in Computer Science to participate. The group saw the draft video, and independently wrote the back-translation of ASL to English on a sheet of paper, then had a group discussion on their

\footnotetext{
${ }^{11}$ In an ASL role shift construction, a signer momentarily becomes a character engaged in a scene with some other imaginary individual. The production of this construction typically involves twisting the torso to one side and aiming the eye
} 
Table 6. List of Back-Translation Studies for ASL-NPS (subset of items listed in Table 1)

\begin{tabular}{|c|l|c|l|}
\hline Study Label & \multicolumn{1}{|c|}{ Type of Study } & \# Particip. & Details of Study \\
\hline $2018-B T-1$ & $\begin{array}{l}\text { ASL-NPS Back-Translation } \\
\text { Round 1 }\end{array}$ & 4 & $\begin{array}{l}\text { Back-translated ASL-NPS to } \\
\text { English, group discussion }\end{array}$ \\
\hline $2018-B T-2$ & $\begin{array}{l}\text { ASL-NPS Back-Translation } \\
\text { Round 2 }\end{array}$ & 12 & $\begin{array}{l}\text { Back-translated ASL-NPS to } \\
\text { English, shared critique with } \\
\text { researcher }\end{array}$ \\
\hline
\end{tabular}

opinion of how the translation could be improved. Our team then went over the feedback we received from the group before producing a final draft version, which took into consideration the subtle changes to the ASL translation that we agreed on. Below is a list of some of the changes the group debated and recommended:

- The draft used the ASL sign INFORM, but the group decided that this word alone didn't convey the meaning of "recommend" in a strong enough way. Instead, the group suggested the idea of using COME-COME in the translation. So, that portion of the translation became: INFORM COME-COME USE THIS down $_{\text {. }}$

- Another aspect the group did not like was the use of the sign MUST as a translation for "extremely likely" as the sign could be perceived as a deontic verb (indicating a duty for someone to share the information with their friend), while the concept being conveyed in the original English NPS question was actually conveying an epistemic modality (simply indicating the likelihood/probability of someone taking some action). While the English modal verb "must" may be used in both senses (e.g., "You must pay your taxes" vs. "You must be Sarah's husband"), the ASL verb MUST primarily conveyed the deontic sense. Therefore, the group recommended that we use FOR SURE INFORM instead of MUST INFORM.

- Finally, while the sign THUMBS-UP was appropriate for conveying approval of something, some members of the group was worried that viewers would perceive it as the ASL sign TEN (consisting of the same thumbs-up handshape in an upright orientation, but with more movement of the hand). The group's concern was that the viewer might interpret this sign as a "suggestion" to answer 10 on the scale. After some discussion, the group recommended use of the sign A-OK ${ }^{12}$ instead. Although A-OK uses the same handshape as the ASL sign NINE, there was less danger of confusion, since the difference in orientation and movement was greater for the pair A-OK and NINE, as compared to the pair THUMBS-UP and TEN.

5.3.1 Second Round of Back-Translation for ASL-NPS. Based on the recommendations of participants in the first round of back-translation, we produced a new version of the ASL-NPS. Appendix E contains a transcript of this final version of the instrument

For the second round (2018-BT-2) of the back-translation, we used the same experimental methodology as described in Section 4.3, with a slight change: since the NPS contained only one question item, we provided participants with 5 SUS questions (items 1-5) as a "warm-up" before giving them the NPS item to back-translate. We recruited 12 participants (10 female, 2 male) from a bachelor's degree program for ASL interpreting at our university (Rochester Institute of Technology) and their ages ranged from 21 to 27 (mean $=22.08$, median $=21$ ), and this pool of interpreters did not participate in the previous back-translation studies for ASL-SUS. Those

gaze at an imaginary person standing to their side whom they are interacting with. During this time, the signer's facial expressions typically convey the emotional state of the character whom they are portraying in the scene.

${ }^{12}$ This is signed with the thumb and index fingers connected to form a circle, with the other fingers straightened. 
participants had been using ASL for 2 to 9 years (mean $=5.25$, median $=4.5$ ). As in the ASL-SUS back-translation studies, those participants watched the ASL-NPS video, wrote an English translation of the question, then provided comments they had about the translation. Participants were paid $\$ 40$ for participating in this one-hour study, which was approved by the university IRB.

Our team analyzed the data, and noticed that many participants (7) managed to write down the word "recommend" even when we did not explicitly use the sign SUGGEST/RECOMMEND, as discussed in Section 5.1. Among participants whose English back-translations of the ASL NPS differed in specific wording from the original NPS instrument, all captured the conceptual meaning of the original question item, if not the specific words. Below, we display some examples of backtranslations produced by participants in the study, along with some of their comments after seeing the original English NPS text (which they could compare to their back-translation):

- P3: "On a scale from 0 to 10, 0 meaning I wouldn't recommend this product/service, 5 meaning possibly, 10 meaning definitely. Where would you rank this product/service in term of recommendations?"

-Comment: "Why scale 0-10? 1 to 10 seems more natural for scaled surveys."

- P10: "Now that you've been through this process, on a scale of 0-10, how likely are you to recommend this to a friend. 0 being not likely and 10 being very likely."

-Comment: "I think 'this process' that I wrote and 'the product' are just a matter of contextual information, but the rest seems to be adequately representing the concept."

- P11: "Now that you have tested this product, how likely are you to encourage a friend to try it as well. On a scale of 0 to 10.0 meaning 'not worth your time,' 5 meaning 'give it a try,' 10 meaning 'absolutely use this product'."

-Comment: "Though the English is different the concept is the same."

The results from the second back-translation user study (2018-BT-2) confirmed that the changes we made in the first round (2018-BT-1) was satisfactory, and our team deemed it unnecessary to revise the ASL-NPS translation any further.

\subsection{Summative User Study Evaluation (Stage 4)}

During the fourth stage, we evaluated our ASL-NPS question, by conducting two studies in which DHH participants evaluated the usability of several artifacts. These two studies were described previously as 2018-US-ASL and 2018-US-ENG, as listed in Table 3. The only difference between the studies was the number of participants (30 in study 2018-US-ASL, 12 in study 2018-US-ENG) and whether they responded to the ASL version of the questionnaires (study 2018-US-ASL) or the original English version (study 2018-US-ENG), after they interacted with the artifacts. We want to remind the reader that Section 4.4 describes the user studies in more detail as the process for ASL-NPS utilized the same user-studies and dataset for analysis.

\subsection{Dissemination of ASL-NPS (Stage 5)}

In the final stage of our study, we have disseminated our new ASL-NPS, to share this resource with the research community. Appendix E provides a transcript of the ASL-NPS, and the final version of the ASL video and PDFs of the paper answer sheets are available at http://latlab.ist.rit. edu/taccess2019asl. Furthermore, please refer to Appendix $\mathrm{H}$ for a description of the entire dataset that is also posted on the ACM Digital Library.

\subsection{ASL-NPS Results}

This section analyzes the results from our evaluation of ASL-NPS, with a focus on addressing the research questions in Section 3.1. 
Our first research question (RQ1) was whether we had preserved the meaning of the original English NPS during the translation process used to produce ASL-NPS. We followed the same methodology as described for ASL-SUS (Section 4). Specifically, the positive results from our second round of back-translation studies strongly suggest that the meaning of the instrument was preserved. Further, without this minimum level of quality, it would be unlikely for the ASL-NPS instrument to succeed at the various psychometric validations discussed below for RQ2.

Informally, we note that the mean scores for the original English NPS (6.188) and the ASLNPS (5.55) were similar ${ }^{13}$; this is a reasonable result, since DHH participants evaluated identical artifacts and performed identical tasks in both studies. A Mann-Whitney U-test was performed to compare the means, and the difference was not significant $(U=2583.5, p=0.2954)$.

In regard to RQ2, Sections 5.6.1 and 5.6.2 analyze the results from studies 2018-US-ASL and 2018US-ENG to examine two key psychometric properties: internal reliability and criterion validity. It is not possible to analyze the construct validity of ASL-NPS via a factor-structure analysis, since the questionnaire contains only one item.

5.6.1 Internal Reliability. Since the NPS has only one question item, we were unable to use Cronbach's Alpha or McDonald's Omega to validate the internal reliability of ASL-NPS, as those methods require at least 2 items in the instrument. After reviewing literature related to evaluating single-item measures $[6,7,79,80]$, we chose to use the single-item "Correction for Attenuation" formula [51] to estimate the reliability for ASL-NPS as it was simple to calculate, given the Alpha value for ASL-SUS (provided in Section 4.6.1) and the correlation between ASL-SUS and ASL-NPS (provided in Section 5.6.2). Following their method (for more details on the formula, see Appendix I), we calculated a reliability score for ASL-NPS of 0.697 . Generally, reliability scores at the 0.7 level and higher are considered reasonable (see Appendix I.3 for more details); so, this result is at the threshold.

5.6.2 Criterion Validity. One method of evaluating criterion validity is to determine how convergent an instrument is with other instruments that measure the same abstract concept. Section 5.4 described how our DHH participants (study 2018-US-ASL), in addition to responding to ASL-NPS, also responded to the ASL-SUS. To assess whether the scores from each participant on the ASL-SUS were correlated to the scores from that participant on the ASL-NPS, we calculated the Pearson's coefficient $r=0.712, p<0.001$. This result indicated a significant correlation ${ }^{14}$ between participants' responses to these instruments.

To assess the criterion validity of ASL-NPS, we compare this correlation above with three other correlations:

- Previously Published Correlation between English SUS and English NPS: In Reference [64], Sauro calculated the correlation between the English SUS and English NPS from two different datasets: Pearson's $r=0.6$ for the 2010 dataset, and $r=0.623$ for the updated dataset. We performed a Fisher's r-to-z transformation on both sets of correlations (ASLNPS-to-ASL-SUS compared to English-NPS-to-English-SUS) and no significant difference was observed: ( $p=0.091, z=1.69)$ for the old dataset and $(p=0.059, z=1.89)$ for the updated dataset.

- Previously Published Correlation between Polish SUS and Polish NPS: In Reference [9], the authors provided the correlation between their participants' Polish SUS scores and their Polish NPS scores: Spearman's $r=0.542, p<0.01$. We performed a Fisher's r-to-z

\footnotetext{
${ }^{13}$ Per-artifact means: (RIT: 8.08 for NPS, 7.37 for ASL-NPS), (Monroe: 3.92 for NPS, 4.57 for ASL-NPS), (Trulia: 7.25 for NPS, 6.40 for ASL-NPS), and (Camera: 5.50 for NPS, 3.87 for ASL-NPS).

${ }^{14}$ We also calculated Spearman's rho: 0.706 with $p<0.001$ for ASL-SUS to ASL-NPS.
} 
transformation ${ }^{15}$ to determine whether this ASL-SUS-to-ASL-NPS correlation was significantly different than the Polish-SUS-to-Polish-NPS correlation reported in [9]; no significant difference was observed ( $p=0.1527, z=1.43$ ).

- Study US-ENG Correlation between English SUS and English NPS: The participants who provided the response data in the above studies $[9,64]$ consisted almost entirely of hearing participants. To compare our study 2018-US-ASL correlation above with data from a more similar group of users, we can examine the data from study 2018-US-ENG, in which $12 \mathrm{DHH}$ participants responded to the original English SUS and to the English NPS. We calculated the correlation between SUS and NPS for these users: Pearson's $r=0.839, p<$ 0.001. To compare our study 2018-US-ASL and study 2018-US-ENG results, a Fisher's $r-$ to-z transformation was performed on the ASL-SUS-to-ASL-NPS correlation to determine whether it was significantly different than the English-SUS-to-English-NPS correlation; no significant difference was observed $(p=0.0615, z=1.87)$.

Thus, we conclude that there is strong evidence that the ASL-NPS has comparable criterion validity to the original English NPS, using the correlation between SUS and NPS as our criterion.

\section{CONCLUSIONS}

The specific goal of this project was to create and evaluate ASL translations of popular usability questionnaires (SUS and NPS), commonly used by HCI researchers and practitioners to quickly evaluate the usability of websites or software. We investigated (Sections 2.3.2 and 2.4) and utilized (Sections 4.1-4.3) accepted methodologies for formally translating a pre-existing English instrument into ASL.

We also investigated (Sections 2.3.3 and 2.3.4) and utilized (Sections 4.4 and 4.6) accepted methodologies for evaluating whether a translated instrument has preserved the meaning of the original version and whether it possesses various psychometric properties (Section 2.2.2). Our results provide strong evidence of the criterion validity of ASL-SUS (as related to the adjective scale of Reference [5]) and of internal reliability (based on a Cronbach's Alpha calculation and alternative methods discussed in Appendix G). Furthermore, the larger set of DHH participant response scores collected in this research (as compared to the smaller dataset in our original conference paper) enabled us to identify strong evidence of the construct validity of ASL-SUS, as evaluated through a detailed analysis of its factor structure, as compared to that of the original English SUS. In summary, the results of our back-translation and our psychometric evaluation of ASL-SUS indicate that this instrument is suitable for use by researchers who wish to evaluate software or websites through studies with DHH participants.

The second instrument we translated, NPS, is not only used in HCI or usability testing contexts, but it is also utilized in wide variety of customer-service or product-evaluation contexts. Our translation of this instrument followed the methodologies established by our original work on ASL-SUS, and it enabled us to investigate the use of an alternate back-translation study design, involving a group discussion, which we found extremely useful, especially for this brief instrument. Our subsequent psychometric analysis of ASL-NPS demonstrated the internal reliability and criterion validity of this translated instrument, and this work has also demonstrated techniques for evaluating these psychometric properties when conducting a translation of a single-item instrument.

\footnotetext{
${ }^{15}$ Siegel [73] described how the Fisher's r-to-z transformation could also be applied to Spearman's rho if the sample size was greater than 10: the Polish study had 37 participants and our 2018-US-ASL study had 30 participants.
} 
The broader goal of this study has been to facilitate the participation of people who are DHH in HCI research studies or in commercial usability testing by HCI practitioners, including during studies that primarily include hearing participants. By making it easier for HCI researchers to provide DHH users with an understandable version of these common usability questionnaires (SUS and NPS), we hope to reduce a barrier for including DHH participants.

Beyond the two specific instruments we have translated for the research community in this work, our intention has been to provide a useful methodological example for future computing accessibility researchers who may wish to translate additional standardized survey instruments into sign language, to promote greater participation and inclusion of people who are DHH in HCI and usability research.

\subsection{Limitations}

While providing ASL versions of standard surveys may address the issue of lower English literacy rates among many deaf individuals that would make English instruments difficult to use, this study has not addressed other potential barriers, including: providing access to low-literacy DHH individuals with weaker ASL skills, helping researchers find and recruit DHH individuals for studies, or supporting communication of task instructions or other information for DHH users that is necessary for participation in the study. Additionally, the DHH participants in our study were mostly university students between ages 20 and 30; for future psychometric evaluation, researchers should also investigate responses gathered from a wider age range of $\mathrm{DHH}$ participants.

\subsection{Future Work}

Our lab regularly conducts usability studies with ASL users, and we intend to continue to make use of the ASL-SUS, ASL-Adj, and ASL-NPS instruments, to enlarge our dataset for additional analyses in the future. While we evaluated several psychometric properties, there are additional properties that should be evaluated in subsequent studies, e.g., external reliability (i.e., whether someone re-taking ASL-SUS on another occasion obtains a similar score). Furthermore, now that our team has gained experience in translating usability questionnaires to ASL and validated the methodology, we plan to undertake a similar project for the CSUQ [40]-or other popular instruments.

While this article has described research into translating these standardized instruments from English into ASL, additional research would be needed to create translations of these surveys that are suitable for use by individuals who are Deaf sign-language users in other countries, where different signed languages are used. While researchers would need to engage in a similar translation process as in our project to produce instruments in other signed languages, some of the translation choices used in our work, e.g., the use of space around the signer's body to represent the item being discussed, may inform the translation of survey questions into other signed languages.

\section{APPENDICES}

\section{A TEXTUAL DESCRIPTION OF FIGURE 1 (TABLE OF CONTENTS FLOWCHART)}

This Appendix will describe Figure 1, the "Table of Contents" flowchart figure in a textual format for readers whom prefer this rather than viewing a figure (such as screen reader users).

The figure is split into two vertical panes, each describing one phase of the project with the left pane titled "Phase 1: System Usability Scale (SUS), Section 4" and the right pane titled "Phase 2: Net Promoter Score (NPS), Section 5." Furthermore, each vertical pane has several sub-panes with a variety of textual content in each of them, arranged by stage (stages 1 to 5) with a big black arrow pointing downwards visually separating each stage. 


\section{A.1 SUS Pane}

The left pane (SUS) begins with Stage 1: "Team of experts translates SUS into ASL and creates a script of the ASL version, after several rounds of exchanging informal videos and discussing drafts (Section 4.1)." Stage 2 is: "A native ASL-signer is recorded performing the SUS instructions, response scale, and question items, with an ASL-signer coach checking that the script is followed (Section 4.2)." Stage 3 is: "Back-translation studies with ASL interpreter students, with revision of ASL-SUS after the first study." There are two more sub-panes in Stage 3, and the first one is: "2017BT-1: First study with 10 participants back-translating ASL-SUS (Section 4.3)" with the second one: "2017-BT-2: Second study with 9 participants back-translating ASL-SUS (Section 4.3.1)."

Stage 4 of SUS has several sub-panes visually aligned in different rows, and the stage begins with a header text: "User-studies with ASL-SUS and ASL-Adj companion instrument (and English SUS and English Adj Scale) to assess psychometric properties of ASL-SUS.” The first row has two sub-panes, and the first one is: "2017-US-ASL: Study with 30 deaf participants using a university website and responding to ASL-SUS and ASL-Adj" and the second one is: "2017-US-ENG: Study with 10 deaf participants using a university website and responding to English SUS and English Adj. Scale." The next row is: "Psychometric analysis of ASL-SUS using data from studies 2017US-ASL and 2017-US-ENG only, which was published in our ASSETS'17 paper, which is citation number 28 in this paper." The next row has two sub-panes, with the first one: "2018-US-ASL: Study with 30 deaf participants using a university website, a government website, a tablet app, and a digital camera, then responding to ASL-SUS and ASL-Adj-as well as to ASL-NPS (Section 4.4.1)" and the second one: "2018-US-ENG: Study with 12 deaf participants using a university website, a government website, a tablet app, and a digital camera, then responding to English SUS and English Adj. Scale-as well as to English NPS (Section 4.4.3)." The final row is: "New psychometric analysis of ASL-SUS using data from studies 2017-US-ASL, 2017-US-ENG, 2018-US-ASL, and 2018-US-ENG; presented in Section 4.6."

The final stage (Stage 5) for SUS is: "Distribute ASL-SUS materials and study data (Section 4.5)."

\section{A.2 NPS Pane}

The right pane (NPS) is visually aligned similarly to the SUS pane with some similar text too, but with different text in Stage 4. Stage 1 is: "Team of experts translates NPS into ASL and creates a script of the ASL version, after several rounds of exchanging informal videos and discussing drafts (Section 5.1)." Stage 2 is: "A native ASL-signer is recorded performing the NPS question item and response scale, with an ASL-signer coach checking that the script is followed (Section 5.2)." Stage 3 is: "Back-translation studies with ASL interpreter students, with revision of ASL-NPS after the first study." There are two more sub-panes in Stage 3, and the first one is: "2018-BT-1: First study with 4 participants back-translating ASL-NPS (Section 5.3)" with the second one: "2018-BT-2: Second study with 12 participants back-translating ASL-NPS (Section 5.3.1)."

Stage 4 of SUS begins with: "User-studies with ASL-NPS (and English NPS, and with use of ASL-SUS as a comparison instrument) to assess psychometric properties of the new ASL-NPS instrument. In addition, these two studies also included ASL or English SUS, to accumulate more response data for the SUS instrument in Phase 1, to enable a new psychometric analysis to be performed for ASL-SUS, with a greater pool of data (discussed in Section 4.4)." The next row has two sub-panes, and the first one is: "2018-US-ASL: Study with 30 deaf participants using a university website, a government website, a tablet app, and a digital camera, then responding to ASL-NPS-as well as to ASL-SUS and ASL-Adj (Section 4.4.1)" and the second one is: "2018-USENG: Study with 12 deaf participants using a university website, a government website, a tablet app, and a digital camera, then responding to English NPS-as well as to English SUS, and English 
Adj. Scale (Section 4.4.3)." The next row has the text: "Note: The data from studies 2018-US-ASL and 2018-US-ENG were used to assess the psychometric properties of ASL-NPS (see below), and to supplement the response data collected for the ASL-SUS (from Phase 1: studies 2017-US-ASL and 2017-US-ENG), to enable a larger, new psychometric analysis of ASL-SUS (see Section 4.6)." The final row in stage 4 is: "Psychometric analysis of ASL-NPS using data from studies 2018-US-ASL and 2018-US-ENG; presented in Section 5.6."

The final stage (Stage 5) for NPS is: "Distribute ASL-NPS materials and study data (Section 5.5)."

\section{B GLOSS CONVENTIONS AND VIDEO AVAILABILITY}

Videos of the ASL version of the SUS question items, Adjective Scale, and NPS are online at this URL: http://latlab.ist.rit.edu/taccess2019asl. Furthermore, the entire collection of videos, answer sheets, and raw participants' numerical responses are also available in the ACM Digital Librarysee Appendix $\mathrm{H}$.

A gloss transcription of each ASL video, using English words to informally represent ASL signs, appears in the subsequent appendices below, for the reader's convenience. We include below some notes on notational conventions used in this appendix and in stage 3 of the translation (Sections 4.3 and 5.3): there are a wide variety of notational conventions used by ASL linguists and interpreters to informally represent ASL signing in a written form; for this article, we have used the following conventions:

- Capital letters will be used to represent individual signs. When multiple English words are being used to represent a single ASL sign, then hyphens will be used, e.g., DON'T-MIND is a single sign in ASL (i.e., right index finger sliding off the nose and then pointing forward away from the body).

- Lowercase letters and braces (i.e., " \{\} ") indicate non-manual aspects of the performance and the span of words during which they occur, e.g., disgust-face-without-head-shake\{ DIFFERENT $\}$ indicates that the signer performs the sign DIFFERENT while simultaneously producing a negative disgust facial expression (without any side-to-side head shaking, which often co-occurs with this facial expression).

- Additional gestural aspects of the performance that are difficult to convey using the above notations are simply indicated with lowercase words in parentheses, e.g., (shrug).

\section{ASL TRANSLATION OF SUS}

The SUS scale was designed in Reference [10]. Prior to the 10 question items, the SUS customarily begins with brief instructions. The ASL translation below differs in content from the English text, since the participant must be instructed to watch videos in the ASL version. In addition, the signer explains the Likert-style response scale for the question items, with options: strongly disagree, disagree, neutral, agree, and strongly agree.

Introduction: English: "Instructions: For each of the following statements, mark one box that best describes your reactions to the website today." ASL Transcript: HELLO, rhetorical\{ THIS QUESTION WHAT \}, SEE THIS PAPER, HAVE SENTENCE. I WILL SIGN EACH SENTENCE. rhetorical\{ YOU DO-DO \} WATCH POINT SENTENCE, WATCH, WONDER YOU AGREE OR DISAGREE, wh-question $\{$ WHICH $\}$. yes-no-question\{ REMEMBER BEFORE \}, YOU PLAY THIS down $_{\text {n }}$ THING, VARIOUS, MAYBE PROGRAM, MAYBE WEBSITE, MAYBE ELECTRIC, DIFFERENT. PLAYING THIS $_{\text {down }}$ TEST. NOW, YOU DREAM-BUBBLE-IMAGINE QUESTION ASL. rhetorical\{ AGREE OR DISAGREE, HOW ANSWER \}. SEE POINT BOX 12334 5, (nodding). rhetorical\{ 1 WHAT $\}$ STRONGLY DISAGREE. rhetorical\{ 2 WHAT $\}$ OKAY DISAGREE. rhetorical\{ 3 WHAT $\}$ 
NEUTRAL. rhetorical\{ 4 WHAT $\}$ OKAY AGREE. rhetorical\{ 5 WHAT $\}$ STRONGLY AGREE. YOU OBSERVE, PICK ONE, CIRCLE ANSWER.

Note: The ASL signer in the video introduction above pointed to a location in the lower region of the signing space to represent the concept of the product, website, or software that the participant had just used. In the transcriptions below, whenever the signer refers to the "product," he points to this region in the signing space again. This avoids the redundancy of the signer repeating the phrase "software, website, or product" for every question item below. This pointing sign is glossed using THIS $_{\text {down }}$ in the transcriptions below.

Q1: English: "I think that I would like to use this product frequently." ASL Transcript: I LOOK THIS $_{\text {down }}$, head-nod-with-mouth-morpheme-cheek-puff DON'T-MIND USE OFTEN $\}$

Q2: English: "I found the product unnecessarily complex." ASL Transcript: I LOOK THIS down, negative-grimace\{ WAVE-WOW COMPLEX

Q3: English: "I thought the product was easy to use." ASL Transcript: I LOOK THIS ${ }_{\text {down }}$, headnod-with-mouth-morpheme-cheek-puff\{ EASY USE UNDERSTAND \}

Q4: English: "I think that I would need the support of a technical person to be able to use this product.” ASL Transcript: I DREAM-BUBBLE-IMAGINE THIS ${ }_{\text {down }}$ USE, I NEED TECHNOLOGY HELP $_{\text {me }}$ (emphatic-nod)

Q5: English: "I found the various functions in the product were well integrated." ASL Transcript:

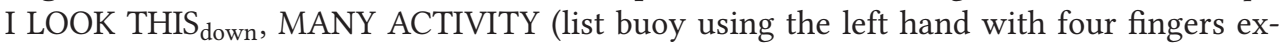
tended to indicate list items, using right index finger to point to each item one-by-one, with rhythmic nodding when pointing to each item) I CAN USE TOGETHER FINE, WAVE-WOW.

Q6: English: "I thought there was too much inconsistency in this product." ASL Transcript: I LOOK THIS $_{\text {down }}$, MANY ACTIVITY (list buoy using the left hand with four fingers extended to indicate list items, using right index finger to point to each item one-by-one, with rhythmic nodding when pointing to each item) disgust-face-without-headshake\{ DIFFERENT \} disgust-face-with-negative-headshake\{ NOT SAME \}

Q7: English: "I imagine that most people would learn to use this product very quickly." ASL Transcript: I DREAM-BUBBLE-IMAGINE LOOK THIS ${ }_{\text {down }}$ LEARN USE FAST (shrug)

Q8: English: "I found the product very awkward to use." ASL Transcript: I LOOK THIS down, I USE disgust-face-without-head-shake-with-tense-grimace\{ AWKWARD \}

Q9: English: "I felt very confident using the product." ASL Transcript: I LOOK THIS down $_{\text {, I USE }}$ happy-positive-face\{ CONFIDENT \}

Q10: English: "I needed to learn a lot of things before I could get going with this product." ASL

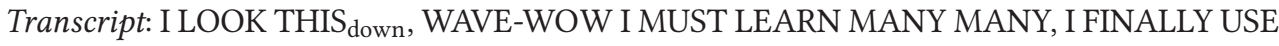

\section{ASL TRANSLATION OF ADJECTIVE SCALE}

The Adjective Scale (Adj) was designed in Reference [5] to be used with the SUS. More details about our usage of this scale is presented in Section 4.4.2. The translation team decided that the terms "user-friendliness" and "product" needed some expansion in ASL to convey the meaning, e.g., to suggest that the person might have seen a website, a computer program, and so on.

English: "Overall, I would rate the user-friendliness of this product as: worst imaginable, awful, poor, OK, good, excellent, best imaginable." ASL Transcript: yes-no-question\{ REMEMBER BEFORE YOU PLAY THIS THING \} VARIOUS, MAYBE PROGRAM, MAYBE WEBSITE, MAYBE ELECTRIC. PLAY, TEST, (nodding). NOW, I CURIOUS, WHAT YOUR OPINION THIS down. $_{\text {. }}$ rhetorical-question\{ HOW EXPLAIN THIS \} yes-no-question\{ SEE BOX (points to first checkbox on paper sheet) \} YOU OBSERVE, I EXPLAIN. FIRST, RIGHT SIDE BOX, NO GOOD 
disgust-face\{ WORST $\}$ NEXT, disgust-face $\{$ AWFUL $\}$ NEXT, disgust-face\{POOR $\}$ NEXT, grimace OK \} NEXT, shrug\{ GOOD \} NEXT, positive-face\{ EXCELLENT \} NEXT, positive-face\{ CHAMP-

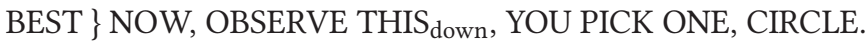

\section{E ASL TRANSLATION OF NPS}

The Net Promoter Score (NPS) was designed in Reference [53]. Similar to the translation for ASLSUS (Appendix C), we modified the wording for "company/product" to generalize it to any artifact (using THIS $_{\text {down }}$ ) under study for HCI researchers to use.

English: "How likely is it that you would recommend our company to a friend or colleague?" ASL Transcript: topic-eyebrow-raise\{ YOU \} cheek-puff-side-release\{ USE \} topic-eyebrow-raise\{ THIS $\left._{\text {down }}\right\}$ FINISH. conditional\{ NOW \} DREAM-BUBBLE-IMAGINE role-shift-gaze-right $\{$ MEET FRIEND POINT $\left._{\text {right }}\right\}$. rhetorical\{ QUESTION WHAT $\}$ THIS $_{\text {down }}$ yes-no-question $\{$ WILL YOU topiceyebrow-raise $\left\{\right.$ THIS $\left._{\text {down }}\right\}$ role-shift-gaze-right INFORM intense-teeth-baring\{ COME-COME \} USE THIS $\left._{\text {down }}\right\}$ QUESTION-MARK-WIGGLE \} topic-eyebrow-raise\{ ANSWER \} rhetorical\{ (slight head shake) $\}$ topic-eyebrow-raise\{ ZERO \} HAND-SWEEP topic-eyebrow-raise\{ TEN \}. topiceyebrow-raise\{ ZERO \} rhetorical\{ (slight head shake) $\}$ head-shake-no\{ REFUSE \} role-shift-gazeright INFORM COME-COME USE \} slight-head-shake\{ THIS ${ }_{\text {down }}$ DISCARD \}. HAND-SWEEP topic-eyebrow-raise\{ FIVE \} MAYBE SO-SO. HAND-SWEEP topic-eyebrow-raise\{ TEN \} headnod-yes FOR SURE \} role-shift-gaze-right\{ INFORM COME-COME USE \} topic-eyebrow-raise\{ THIS $\left._{\text {down }}\right\}$ check-puff-side-release $\{\mathrm{A}-\mathrm{OK}\}$.

\section{F STUDY ARTIFACTS AND TASKS}

During our user studies (2018-US-ASL and 2018-US-ENG), we asked participants to interact with four artifacts, and we created several tasks for each of them. Below is a list of tasks for each artifact:

- Our university's website [RIT]: https://www.rit.edu

(1) What events are happening around campus on March 29, 2018?

(2) Find the phone number for the student employment office.

(3) What is the cover title of the Fall/Winter 2017 "Research at RIT" magazine?

(4) How can you make an appointment at the Fitness Lab?

- Our local county government's website [MC]: https://www.monroecounty.gov

(1) What levels of food worker certification does Monroe County recognize?

(2) What are the minimum qualifications for the "Rehabilitation Counselor" Civil Service Exam for the public, which will be provided on May 12, 2018?

(3) What is the fee for recycling flat screen televisions and monitors at the ecopark?

- The "Trulia" Android app [TRU]: https://play.google.com/store/apps/details?id=com.trulia. android

(1) Find a rental in Rochester, NY. Pick any property that allows dogs. Where is the nearest elementary school?

(2) Find a condo for sale in Houston, TX. Pick any property selling for less than $\$ 500,000$. What is the estimated monthly property taxes?

- The LLION 2.7” HD Mini Digital Camera [CAM]: https://www.amazon.com/gp/product/ B077XM7TXM

(1) Take a few pictures with different zoom levels. Record a movie.

(2) Set the self-timer for $\mathbf{5 s}$, then take a selfie :)

(3) Change the settings so the camera takes pictures at 7M HD "Image Size" and set the "Scene" to Sport. Take a picture without the self-timer.

(4) Format the SD card and reset to default settings. 


\section{G ADDITIONAL METHODS OF DEMONSTRATING ASL-SUS INTERNAL RELIABILITY}

As discussed in Section 4.6.1, since previous SUS translation efforts have not provided any additional calculations of internal reliability other than the Alpha, we still chose to present Alpha scores in this article. However, we are providing the results from several alternative methods (as suggested by Revelle and Zinbarg in Reference [58]) to calculate internal reliability of ASL-SUS in Table 7: check the row labelled "ALL". A short description of each method is presented below, refer to [58] for an in-depth analysis of each method and the rationale for selecting McDonald's Omega (Total) as the superior measure of reliability compared to the Alpha.

We calculated McDonald's Omega (Total) [48] for ASL-SUS: $\omega_{\text {total }}=0.918$. McDonald also provided an alternative method [49] to calculate the Omega (Hierarchical): $\omega_{\mathrm{h}}=0.715$ (we note that this is borderline, because this metric requires three factors or more-see Section 4.6.3 for a discussion of the two-factor structure of ASL-SUS). Guttman's Lambda 6 (squared multiple correlation or smc) [28] is another method to estimate the variance in the responses: $\lambda_{6}=0.911$. Revelle suggested the Beta (minimum split half reliability) [58] as a method of calculating the lower bound of reliability: $\beta=0.782$. Guttman's Lambda 4 (maximum split half reliability) [28] is a measure of the upper bound of reliability: $\lambda_{4}=0.939$. After calculating all of the suggested alternative methods, the results show that the reliability of ASL-SUS was consistently excellent (greater than 0.9) and the lower bound $(\beta)$ is considered good (almost 0.8 ).

In our literature review for modern psychometric methods, we found an interesting paper from Lewis that discussed the concept of "leave one out" for the SUS [43]. In his work, he showed that dropping an item resulted in no adverse changes to the reliability of SUS, and we wanted to replicate his work to check whether ASL-SUS held the same property. Shown in Table 7 is the reliability calculation for ASL-SUS, each row such as "Q1" represents the reliability of ASL-SUS with that question item excluded from the calculation. With the prior discussion revealing that McDonald's $\omega_{\text {total }}$ should be the metric we base our results on, the "leave one out" analysis reveals that ASL-SUS consistently scored above 0.9 , and no single question item adversely impacted the overall internal reliability (in comparison to Lewis' work, the Alpha also showed no adverse changes either).

Finally, as reported in the English-to-Portuguese SUS translation [47], the authors used the Intraclass Correlation Coefficient (ICC) to compute an additional measure of inter-rater reliability; however, their reported ICC value was "poor" $(<0.4$ when referencing the table provided

Table 7. Measures of Reliability for ASL-SUS Question Items (Leave One Out)

\begin{tabular}{|l|c|c|c|c|c|c|}
\hline Item & $\begin{array}{c}\text { Cronbach's } \\
\text { Alpha }\end{array}$ & $\begin{array}{c}\text { McDonald's } \\
\omega_{\text {hierarchical }}\end{array}$ & $\begin{array}{c}\text { McDonald's } \\
\omega_{\text {total }}\end{array}$ & $\begin{array}{c}\text { Guttman's } \\
\boldsymbol{\lambda}_{6}\end{array}$ & $\begin{array}{c}\text { Revelle's } \\
\boldsymbol{\beta}\end{array}$ & $\begin{array}{c}\text { Guttman's } \\
\boldsymbol{\lambda}_{4}\end{array}$ \\
\hline ALL & 0.898 & 0.715 & 0.918 & 0.911 & 0.782 & 0.939 \\
\hline Q1 & 0.894 & 0.758 & 0.915 & 0.904 & 0.810 & 0.926 \\
\hline Q2 & 0.879 & 0.693 & 0.903 & 0.891 & 0.769 & 0.914 \\
\hline Q3 & 0.879 & 0.677 & 0.903 & 0.892 & 0.734 & 0.921 \\
\hline Q4 & 0.888 & 0.686 & 0.910 & 0.898 & 0.798 & 0.921 \\
\hline Q5 & 0.895 & 0.756 & 0.914 & 0.902 & 0.792 & 0.917 \\
\hline Q6 & 0.903 & 0.724 & 0.923 & 0.914 & 0.791 & 0.929 \\
\hline Q7 & 0.892 & 0.675 & 0.915 & 0.903 & 0.770 & 0.927 \\
\hline Q8 & 0.880 & 0.686 & 0.904 & 0.894 & 0.781 & 0.918 \\
\hline Q9 & 0.880 & 0.660 & 0.905 & 0.893 & 0.743 & 0.918 \\
\hline Q10 & 0.892 & 0.718 & 0.914 & 0.905 & 0.793 & 0.925 \\
\hline
\end{tabular}


in Reference [14]). It was not clear whether the authors used the correct ICC model $(1 / 2 / 3$ as defined by Shrout and Fleiss [72]) nor utilized the single/average method of computing inter-rater agreement/consistency. While the above reliability calculations already showed a strong result for ASL-SUS, we are providing our ICC values for future reference. Following the advice in Reference [35], we are reporting the two-way random-effects based on a mean-rating model (ICC2k). The ICC for ASL-SUS was 0.839 for agreement with 95\% CI [0.683, 0.949] and 0.908 for consistency with $95 \%$ CI [0.804, 0.972]. Based on the guidelines in Reference [14] for interpreting the ICC, ASL-SUS had "moderate to good" inter-rater reliability.

We conclude that the ASL-SUS has excellent internal reliability compared to SUS using Cronbach's Alpha, McDonald's Omega, and leave-one-out analysis as our criterion.

\section{H USER STUDY DATA (ONLINE APPENDIX)}

This appendix describes the raw data collected in study 2018-US-ASL and study 2018-US-ENG, described in Section 4.4. Since the dataset would be too large to include in the print version, we have included it in the "Online Appendix" for this article in the ACM Digital Library. Readers may also view the supplementary materials at our laboratory website: http://latlab.ist.rit.edu/taccess2019asl. The online appendix also includes the participant answer forms we used in the US-ASL study "participant_sheet-ASL.pdf," the US-ENG study "participant_sheet-ENG.pdf," and videos of the ASL translations of SUS, Adjective Scale, and NPS.

Interested readers are encouraged to visit the ACM Digital Library or the above URL to download the CSV files, and the list (below) describes the contents of the dataset. We want to remind readers that the US-ASL studies utilized the ASL versions of the questionnaires (ASL-SUS, ASLAdj, and ASL-NPS), while US-ENG studies used the original English forms with no ASL instruction. Therefore, the "TACCESS_Berke_dataset_ASL.csv" file corresponds to study US-ASL while the "TACCESS_Berke_dataset_Eng.csv" file corresponds to study US-ENG.

Again, we want to remind readers that this dataset is a concatenation of our older dataset (studies 2017-US-ASL and 2017-US-ENG) that was published (the prior study labeled them \#1 and \#2, respectively) in the ASSETS'17 conference [31], along with data from additional participants in the new user studies (2018-US-ASL and 2018-US-ENG). For the reader's convenience, we have labeled the participants' IDs from the new studies at an offset relative to the older studies. For example: 1 corresponds to participant\#1 from the old ASL study (2017-US-ASL), 81 corresponds to participant\#1 from the new ASL study (2018-US-ASL), 41 corresponds to participant\#1 from the old ENG study (2017-US-ENG), and 61 corresponds to participant\#1 from the new ENG study (2018-US-ENG). You will also notice that all participants from the older studies (2017-US-ASL and 2017-US-ENG) evaluated only one artifact: the RIT website, and they were not asked to respond to the NPS instrument (thus, empty cells in the CSV files for these individuals).

The CSV files contain the following columns:

- "\#" => a code number used to refer to each participant.

- "A" => the artifact the participant evaluated: RIT [the Rochester Institute of Technology webpage at https://www.rit.edu], MC [the Monroe County government webpage at https://www.monroecounty.gov], TRU [the Trulia app on Android: https://play.google.com/ store/apps/details?id=com.trulia.android\&hl=en], and CAM [LLION HD Mini Digital Camera: https://www.amazon.com/gp/product/B077XM7TXM].

- "Q1" to "Q10" => the raw scores from participant responses to individual SUS Likert-type items, with 1 indicating "Strongly Disagree" and 5 indicating "Strongly Agree."

- "SUS" => the overall SUS score based on these ten responses. 
- "Adj" => indicating the numerical values corresponding to the adjective choice of the participant on this artifact-Worst Imaginable (1), Awful (2), Poor (3), OK (4), Good (5), Excellent (6), Best Imaginable (7).

- "NPS" => the raw scores from participant responses that ranged from 0 indicating "Not at all likely" to 10 indicating "Extremely likely."

Because half of the individual items on SUS have negative polarity, the overall SUS score is calculated according to this formula, from Reference [10]:

$$
\begin{aligned}
& \mathrm{SUS}=2.5 *[(\mathrm{Q} 1-1)+(5-\mathrm{Q} 2)+(\mathrm{Q} 3-1)+(5-\mathrm{Q} 4)+(\mathrm{Q} 5-1)+(5-\mathrm{Q} 6)+(\mathrm{Q} 7-1)+(5- \\
& \mathrm{Q} 8)+(\mathrm{Q} 9-1)+(5-\mathrm{Q} 10)] .
\end{aligned}
$$

\section{DESCRIPTION OF THE CORRECTION FOR ATTENUATION FORMULA}

This appendix describes the formula in detail, and this formula is applied in Section 5.6.1.

\section{I.1 Correction for Attenuation (CfA)}

In Psychology textbooks such as Reference [51], the Correction for Attenuation (henceforth shortened to CfA for brevity) formula is described as an approach used to approximate the relation between the rankings of two factors (correlation) while also taking into account errors in the measurements. More specifically, Spearman [75, p. 89] mentioned: "But here in correlations, they also have this new effect, which is always in the direction of 'attenuating' the apparent correspondence and whose amount, depending solely on the size of the middle error, cannot be in the least eliminated by any prolongation of the series." By correcting for this attenuation (disattenuation), estimates of correlation between factors will appropriately account for the errors contained in the variables' measurements.

In our case, we wanted to eliminate the errors in the measurements of the artifacts themselves, and calculate the reliability of ASL-NPS (see Section 5.6.1) via this method, which was shown to work on single-item measures $[6,7,79,80]$, wherein traditional approaches such as Cronbach's Alpha [16] require at least two items in a measure to calculate the resultant value.

\section{I.2 Calculating the CfA Value}

The CfA formula is [80]

$$
\text { CfA }=\frac{\text { correlation of underlying construct between both factors }}{\sqrt{\text { reliability of factor } 1 * \text { reliability of factor } 2}} .
$$

As explained by Bergkvist, performing some arithmetic on the original formula provided by Spearman allows us to estimate the reliability of factor 2 (in our case, the single-item NPS) when we already know the reliability of factor 1 (the SUS) via:

"The correlation between the two measures and the internal consistency reliability (alpha) for the multiple-item measure, which can both be estimated from the data, can be plugged into the formula for correction of attenuation, which leaves the reliability for the single-item measure as the only unknown in the formula." [6, p. 248]

Since we know that the NPS and SUS both attempt to measure similar constructs (recommendation vs. usability) within the same conceptual domain (artifact), it was necessary to select a correlation value as an input parameter to the CfA formula, to perform this reliability analysis. Following standard practice $[79,80]$, we have assumed a moderate correlation (0.9) for the underlying construct, and we used this parameter value in the CfA formula to calculate a reliability 
score for ASL-NPS of 0.697 (we inform the reader that the Bergkvist paper [6, p. 249] used different notation than the Wanous and Hudy paper [79, p. 363]: " $r_{11}$ and $r_{22}$ " compared to " $r_{x x}$ and $r_{y y}$ "):

$$
\begin{aligned}
& r_{12}\left(r_{x y}\right)=\text { correlation of underlying construct }=0.9, \\
& r_{11}\left(r_{x x}\right)=\text { alpha(ASL-SUS) [Section 4.6.1] }=0.898, \\
& r_{22}\left(r_{y y}\right)=\text { cor(ASL-NPS, ASL-SUS) [Section 5.6.2] }=0.712, \\
\text { CfA }= & \hat{r}_{12}\left(r_{x y}\right)=\text { Equation }(1)=\frac{r_{12}}{\sqrt{r_{11} * r_{22}}}=\frac{0.9}{\sqrt{0.898 * 0.712}}=0.697 .
\end{aligned}
$$

The reader should note that assuming a perfect correlation (setting $r_{12}$ to 1.0 ) is generally not done during this type of analysis, and that making such an extreme assumption for that parameter would have resulted in a reliability score of 0.565 , and relaxing this correlation parameter to 0.95 would have resulted in a reliability score of 0.626 .

\section{I.3 Interpreting the CfA}

Generally, reliability values above 0.9 is considered excellent (for example, see Reference [37] for Cronbach's Alpha [16]); however, in our case (CfA): If the value is at least 0.7, then the reliability is considered "reasonable" $[79,81]$, which the authors use to indicate a good result, rather than using the term "excellent," as in the Cronbach's Alpha literature. Another overlooked aspect of CfA is that it actually is an estimate of the minimum reliability of a single-item measure, as Wanous and Hudy said it well:

"A second reason why the two methods appear to disagree is that the correction for attenuation formula produces an estimate of the minimum single-item reliability. Thus, the 'true' reliability could be, and probably is, higher than the estimated minimum.” [79, p. 370]

In conclusion, the true reliability of ASL-NPS would likely be higher; so, the result of this analysis provides strong evidence that the internal reliability of ASL-NPS (0.697) is within this "reasonable" range.

\section{ACKNOWLEDGMENTS}

The authors thank our research assistants, Peter Yeung and Abraham Glasser, for their help in recruiting participants for the user studies. We also want to express our gratitude to many individuals who participated in the back-translation and user studies for this project.

\section{REFERENCES}

[1] Sedeeq Al-khazraji, Larwan Berke, Sushant Kafle, Peter Yeung, and Matt Huenerfauth. 2018. Modeling the speed and timing of American Sign Language to generate realistic animations. In Proceedings of the 20th International ACM SIGACCESS Conference on Computers and Accessibility (ASSETS'18). ACM, New York, NY. DOI : https://doi.org/10.1145/ 3234695.3236356

[2] Bareeq A. AlGhannam, Sana A. Albustan, Abeer A. Al-Hassan, and Lamees A. Albustan. 2017. Towards a Standard Arabic system usability scale: Psychometric evaluation using communication disorder app. Int. f. Hum.-Comput. Interact. 0, 0 (2017), 1-6. DOI : https://doi.org/10.1080/10447318.2017.1388099

[3] American Educational Research Association, American Psychological Association, and National Council on Measurement in Education. 1999. Standards for Educational and Psychological Testing. AERA, Washington, DC.

[4] Aaron Bangor, Philip T. Kortum, and James T. Miller. 2008. An empirical evaluation of the system usability scale. Int. 7. Hum.-Comput. Interact. 24, 6 (2008), 574-594.

[5] Aaron Bangor, Philip T. Kortum, and James T. Miller. 2009. Determining what individual SUS scores mean: Adding an adjective rating scale. 7 . Usabil. Studies 4, 3 (2009), 114-123.

[6] Lars Bergkvist. 2015. Appropriate use of single-item measures is here to stay. Market. Lett. 26, 3 (Sep. 2015), 245-255. DOI : https://doi.org/10.1007/s11002-014-9325-y 
[7] Lars Bergkvist and John R. Rossiter. 2007. The predictive validity of multiple-item versus single-item measures of the same constructs. F. Market. Res. 44, 2 (2007), 175-184. Retrieved from http://www.jstor.org/stable/30162466.

[8] Bojan Blažica and James R. Lewis. 2015. A Slovene translation of the system usability scale: The SUS-SI. Int. F. Hum.Comput. Interact. 31, 2 (2015), 112-117. DOI : https://doi.org/10.1080/10447318.2014.986634

[9] Anna Borkowska and Katarzyna Jach. 2017. Pre-testing of Polish translation of system usability scale (SUS). In Proceedings of the 37th International Conference on Information Systems Architecture and Technology (ISAT'16), Leszek Borzemski, Adam Grzech, Jerzy Świątek, and Zofia Wilimowska (Eds.). Springer International Publishing, Cham, 143-153. DOI : https://doi.org/10.1007/978-3-319-46583-8_12

[10] John Brooke. 1996. SUS-A quick and dirty usability scale. Usabil. Eval. Industry 189, 194 (1996), 4-7.

[11] John Brooke. 2013. SUS: A retrospective. F. Usabil. Studies 8, 2 (Feb. 2013), 29-40. http://dl.acm.org/citation.cfm?id= 2817912.2817913

[12] Barbara S. Chaparro. 2008. Usability evaluation of a university portal website. Usabil. News 10, 2 (2008), 1-7. Retrieved from https://web.archive.org/web/20170124205151http://usabilitynews.org/usability-evaluation-of-a-universityportal-website/.

[13] Eunseong Cho and Seonghoon Kim. 2014. Cronbach's coefficient alpha: Well known but poorly understood. Organiz. Res. Methods 18, 2 (2014), 207-230. DOI : https://doi.org/10.1177/1094428114555994

[14] Domenic V. Cicchetti. 1994. Guidelines, criteria, and rules of thumb for evaluating normed and standardized assessment instruments in psychology. Psychol. Assess. 6, 4 (1994), 284-290. DOI : https://doi.org/10.1037/1040-3590.6.4.284

[15] Anna B. Costello and Jason W. Osborne. 2005. Best practices in exploratory factor analysis: Four recommendations for getting the most from your analysis. Pract. Assess. Res. Eval. 10, 7 (2005), 1-9. Retrieved from http://pareonline. net/getvn.asp?v=10\&n=7.

[16] Lee J. Cronbach. 1951. Coefficient alpha and the internal structure of tests. Psychometrika 16, 3 (1951), $297-334$. DOI : https://doi.org/1010.1007/BF02310555

[17] Aline da Silva Alves, Simone Bacellar Leal Ferreira, Viviane Santos de Oliveira, and Denis S. da Silva. 2012. Evaluation of potential communication breakdowns in the interaction of the deaf in corporate information systems on the web. Procedia Comput. Sci. 14 (2012), 234-244. DOI : https://doi.org/10.1016/j.procs.2012.10.027

[18] Fons J. R. Van de Vijver and Kwok Leung. 1997. Methods and Data Analysis for Cross-cultural Research, vol. 1. Sage Publications.

[19] Iman Dianat, Zahra Ghanbari, and Mohammad AsghariJafarabadi. 2014. Psychometric properties of the Persian language version of the system usability scale. Health Promot. Perspect. 4, 1 (2014), 82-89. DOI : https://doi.org/10.5681/ hpp.2014.011

[20] Mark Dilsizian, Zhiqiang Tang, Dimitris Metaxas, Matt Huenerfauth, and Carol Neidle. 2016. The importance of 3D motion trajectories for computer-based sign recognition. In Proceedings of the 7th Workshop on the Representation and Processing of Sign Languages: Corpus Mining, The 10th International Conference on Language Resources and Evaluation (LREC'16). ELRA, Paris, 53-58.

[21] Thomas J. Dunn, Thom Baguley, and Vivienne Brunsden. 2013. From alpha to omega: A practical solution to the pervasive problem of internal consistency estimation. Brit. f. Psychol. 105, 3 (2013), 399-412. DOI : https://doi.org/10. 1111/bjop.12046

[22] Sarah Ebling and John Glauert. 2016. Building a Swiss German Sign Language avatar with JASigning and evaluating it among the Deaf community. Univ. Access Info. Soc. 15, 4 (Nov. 2016), 577-587. DOI : https://doi.org/10.1007/ s10209-015-0408-1

[23] Oğuzhan Erdinç and James R. Lewis. 2013. Psychometric evaluation of the T-CSUQ: The Turkish version of the computer system usability questionnaire. Int. J. Hum.-Comput. Interact. 29, 5 (2013), 319-326. DOI : https://doi.org/10. 1080/10447318.2012.711702

[24] Kraig Finstad. 2006. The system usability scale and non-native English speakers. f. Usabil. Studies 1, 4 (Aug. 2006), 185-188. http://dl.acm.org/citation.cfm?id=2835531.2835535

[25] Kraig Finstad. 2010. The usability metric for user experience. Interact. Comput. 22, 5 (2010), 323-327. DOI : https:// doi.org/10.1016/j.intcom.2010.04.004

[26] Kurt F. Geisinger. 1994. Cross-cultural normative assessment: Translation and adaptation issues influencing the normative interpretation of assessment instruments. Psychol. Assess. 6, 4 (1994), 304-312.

[27] Patrick Graybill, Julia Aggas, Robyn K. Dean, Susan Demers, Elizabeth G. Finigan, and Robert Q. Pollard Jr. 2010. A community-participatory approach to adapting survey items for deaf individuals and American Sign Language. Field Methods 22, 4 (2010), 429-448.

[28] Louis Guttman. 1945. A basis for analyzing test-retest reliability. Psychometrika 10, 4 (Dec. 1945), 255-282. DOI : https://doi.org/10.1007/bf02288892

[29] Matt Huenerfauth. 2008. Evaluation of a psycholinguistically motivated timing model for animations of American Sign Language. In Proceedings of the 10th International ACM SIGACCESS Conference on Computers and Accessibility (ASSETS’08). ACM, New York, NY, 129-136. DOI : https://doi.org/10.1145/1414471.1414496 
[30] Matt Huenerfauth and Hernisa Kacorri. 2014. Release of experimental stimuli and questions for evaluating facial expressions in animations of American Sign Language. In Proceedings of the 6th Workshop on the Representation and Processing of Sign Languages: Beyond the Manual Channel and the 9th International Conference on Language Resources and Evaluation (LREC'14). ELRA, Paris, 6.

[31] Matt Huenerfauth, Kasmira Patel, and Larwan Berke. 2017. Design and psychometric evaluation of an American Sign Language translation of the system usability scale. In Proceedings of the 19th International ACM SIGACCESS Conference on Computers and Accessibility (ASSETS'17). ACM, New York, NY, 175-184. DOI : https://doi.org/10.1145/ 3132525.3132540

[32] Hernisa Kacorri, Matt Huenerfauth, Sarah Ebling, Kasmira Patel, Kellie Menzies, and Mackenzie Willard. 2017. Regression analysis of demographic and technology-experience factors influencing acceptance of sign language animation. ACM Trans. Access. Comput. 10, 1, Article 3 (Apr. 2017). DOI : https://doi.org/10.1145/3046787

[33] Saba Kawas, George Karalis, Tzu Wen, and Richard E. Ladner. 2016. Improving real-time captioning experiences for deaf and hard of hearing students. In Proceedings of the 18th International ACM SIGACCESS Conference on Computers and Accessibility (ASSETS'16). ACM, New York, NY, 15-23. DOI : https://doi.org/10.1145/2982142.2982164

[34] Rex B. Kline. 2015. Principles and Practice of Structural Equation Modeling (4th ed.). Guilford Press, New York, NY.

[35] Terry K. Koo and Mae Y. Li. 2016. A guideline of selecting and reporting intraclass correlation coefficients for reliability research. f. Chiroprac. Med. 15, 2 (June 2016), 155-163. DOI : https://doi.org/10.1016/j.jcm.2016.02.012

[36] Paddy Ladd. 2003. Understanding Deaf Culture: In Search of Deafhood. Multilingual Matters, Bristol, UK.

[37] Thomas K. Landauer. 1997. Behavioral research methods in human-computer interaction. In Handbook of HumanComputer Interaction (2nd ed.), Marting G. Helander, Thomas K. Landauer, and Prasad V. Prabhu (Eds.). NorthHolland, Amsterdam, 203-227. DOI : https://doi.org/10.1016/B978-044481862-1.50075-3

[38] Jonathan Lazar, Jinjuan Heidi Feng, and Harry Hochheiser. 2017. Research Methods in Human-Computer Interaction (2nd ed.). Morgan Kaufmann, San Francisco, CA.

[39] James R. Lewis. 1991. Psychometric evaluation of an after-scenario questionnaire for computer usability studies: The ASQ. ACM SIGCHI Bull. 23, 1 (Jan. 1991), 78-81. DOI : https://doi.org/10.1145/122672.122692

[40] James R. Lewis. 1995. IBM computer usability satisfaction questionnaires: Psychometric evaluation and instructions for use. Int. J. Hum.-Comput. Interact. 7, 1 (1995), 57-78. DOI : https://doi.org/10.1080/10447319509526110

[41] James R. Lewis. 2002. Psychometric evaluation of the PSSUQ using data from five years of usability studies. Int. $\mathcal{F}$. Hum.-Comput. Interact. 14, 3-4 (2002), 463-488. DOI : https://doi.org/10.1080/10447318.2002.9669130

[42] James R. Lewis and Jeff Sauro. 2009. The factor structure of the system usability scale. In Human Centered Design, Masaaki Kurosu (Ed.). Springer, Berlin, 94-103. DOI : https://doi.org/10.1007/978-3-642-02806-9_12

[43] James R. Lewis and Jeff Sauro. 2017. Can I leave this one out? The effect of dropping an item from the SUS. F. Usabil. Studies 13, 1 (Nov. 2017), 38-46.

[44] James R. Lewis, Brian S. Utesch, and Deborah E. Maher. 2013. UMUX-LITE: When there's no time for the SUS. In Proceedings of the SIGCHI Conference on Human Factors in Computing Systems (CHI'13). ACM, New York, NY, 20992102. DOI : https://doi.org/10.1145/2470654.2481287

[45] Kris Lohmann and Jörg Schäffer. 2013. System Usability Scale (SUS)-An Improved German Translation of the Questionnaire. Retrieved from https://web.archive.org/web/20160330062546http://minds.coremedia.com/2013/09/18/ sus-scale-an-improved-german-translation-questionnaire.

[46] Urbano Lorenzo-Seva and Jos M. F. Ten Berge. 2006. Tucker's congruence coefficient as a meaningful index of factor similarity. Methodol.: Eur. F. Res. Methods Behav. Soc. Sci. 2, 2 (Jan. 2006), 57-64. DOI : https://doi.org/10.1027/1614-2241. 2.2 .57

[47] Ana Isabel Martins, Ana Filipa Rosa, Alexandra Queirós, Anabela Silva, and Nelson Pacheco Rocha. 2015. European portuguese validation of the system usability scale (SUS). Proced. Comput. Sci. 67 (2015), 293-300. DOI : https://doi.org/ 10.1016/j.procs.2015.09.273 In Proceedings of the 6th International Conference on Software Development and Technologies for Enhancing Accessibility and Fighting Info-exclusion (DSAI).

[48] Roderick P. McDonald. 1978. Generalizability in factorable domains: "Domain validity and generalizability." Educat. Psychol. Measure. 38, 1 (1978), 75-79. DOI : https://doi.org/10.1177/001316447803800111

[49] Roderick P. McDonald. 1999. Test Theory: A Unified Treatment. Erlbaum, Hillsdale, NJ.

[50] Ross E. Mitchell, Travas A. Young, Bellamie Bachleda, and Michael A. Karchmer. 2006. How many people use ASL in the United States? Why estimates need updating. Sign Lang. Studies 6, 3 (2006), 306-335.

[51] Jum C. Nunnally and Ira H. Bernstein. 1994. Psychometric Theory (3 ed.). McGraw-Hill, New York, NY.

[52] R Core Team. 2017. R: A Language and Environment for Statistical Computing, R version 3.3.3. R Foundation for Statistical Computing, Vienna, Austria. Retrieved from https://www.R-project.org/.

[53] Frederick F. Reichheld. 2003. The one number you need to grow. Harvard Bus. Rev. 81, 12 (2003), 46-55.

[54] Frederick F. Reichheld. 2006. The microeconomics of customer relationships. MIT Sloan Manage. Rev. 47, 2 (Winter 2006), 73 . 
[55] Frederick F. Reichheld and Rob Markey. 2011. The Ultimate Question 2.0: How Net Promoter Companies Thrive in a Customer-driven World. Harvard Business Publishing, Boston, MA.

[56] William Revelle. 2017. Psych: Procedures for Psychological, Psychometric, and Personality Research, R package version 1.7.8. Northwestern University, Evanston, IL. Retrieved from https:/CRAN.R-project.org/package=psych.

[57] William Revelle and Thomas Rocklin. 1979. Very simple structure: An alternative procedure for estimating the optimal number of interpretable factors. Multivar. Behav. Res. 14, 4 (1979), 403-414. DOI: https://doi.org/10.1207/ s15327906mbr1404_2

[58] William Revelle and Richard E. Zinbarg. 2008. Coefficients alpha, beta, omega, and the glb: Comments on Sijtsma. Psychometrika 74, 1 (11 Dec 2008), 145. DOI : https://doi.org/10.1007/s11336-008-9102-z

[59] Yves Rosseel. 2012. lavaan: An R package for structural equation modeling. F. Stat. Softw. 48, 2 (2012), 1-36. DOI : https: //doi.org/10.18637/jss.v048.i02

[60] Bernard Rummel. 2015. System Usability Scale-Jetzt auch auf Deutsch. Retrieved from https://experience.sap.com/ skillup/system-usability-scale-jetzt-auch-auf-deutsch/.

[61] John K. Sakaluk and Stephen D. Short. 2017. A methodological review of exploratory factor analysis in sexuality research: Used practices, best practices, and data analysis resources. F. Sex Res. 54, 1 (2017), 1-9. DOI : https://doi.org/ 10.1080/00224499.2015.1137538

[62] Waheedy Samady, Georgia Robins Sadler, Melanie Nakaji, and Vanessa L. Malcarne. 2008. Translation of the multidimensional health locus of control scales for users of American Sign Language. Public Health Nurs. 25, 5 (2008), 480-489. DOI : https://doi.org/10.1111/j.1525-1446.2008.00732.x

[63] Jeff Sauro. 2010. 5 Second Usability Tests. Retrieved from https://measuringu.com/five-second-tests/.

[64] Jeff Sauro. 2012. Predicting Net Promoter Scores from System Usability Scale Scores. Retrieved from https:// measuringu.com/nps-sus/.

[65] Jeff Sauro and James R. Lewis. 2011. When designing usability questionnaires, does it hurt to be positive? In Proceedings of the SIGCHI Conference on Human Factors in Computing Systems (CHI'11). ACM, New York, NY, 2215-2224. DOI : https://doi.org/10.1145/1978942.1979266

[66] Jeff Sauro and James R. Lewis. 2016. Quantifying the User Experience: Practical Statistics for User Research (2nd ed.). Morgan Kaufmann, Cambridge, MA.

[67] Jerry Schnepp and Brent Shiver. 2011. Improving deaf accessibility in remote usability testing. In Proceedings of the 13th International ACM SIGACCESS Conference on Computers and Accessibility (ASSETS'11). ACM, New York, NY, 255-256. DOI : https://doi.org/10.1145/2049536.2049594

[68] Andrew Sears and Vicki Hanson. 2011. Representing users in accessibility research. In Proceedings of the ACM SIGCHI Conference on Human Factors in Computing Systems (CHI'11). ACM, New York, NY, 2235-2238. DOI : https://doi.org/ $10.1145 / 1978942.1979268$

[69] Sanjay Seth, Don Scott, Chad Svihel, and Stephen Murphy-Shigematsu. 2016. Solving the mystery of consistent negative/low net promoter score (NPS) in cross-cultural marketing research. Asia Market. f. 17, 4 (2016), 43-61. DOI : https://doi.org/10.15830/amj.2016.17.4.43

[70] Zahra Sharfina and Harry Budi Santoso. 2016. An Indonesian adaptation of the system usability scale (SUS). In Proceedings of the International Conference on Advanced Computer Science and Information Systems (ICACSIS'16). 145-148. DOI : https://doi.org/10.1109/ICACSIS.2016.7872776

[71] Feng-Ru Sheu, Hui-Jung Fub, and Meilun Shih. 2017. Pre-testing the Chinese version of the system usability scale (CSUS). In Proceedings of the 25th International Conference on Computers in Education-Workshop W1: The Applications of Information and Communication Technologies in Adult and Continuing Education (ICCE'17).

[72] Patrick E. Shrout and Joseph L. Fleiss. 1979. Intraclass correlations: Uses in assessing rater reliability. Psychol. Bull. 86, 2 (1979), 420-428. DOI : https://doi.org/10.1037/0033-2909.86.2.420

[73] Sidney Siegel. 1956. Non-parametric Statistics for the Behavioral Sciences. McGraw-Hill Book Co., Inc., New York, NY.

[74] Klaas Sijtsma. 2008. On the use, the misuse, and the very limited usefulness of Cronbach's alpha. Psychometrika 74, 1 (11 Dec 2008), 107. DOI: https://doi.org/10.1007/s11336-008-9101-0

[75] C. Spearman. 1904. The proof and measurement of association between two things. Amer. F. Psychol. 15, 1 (1904), 72-101. http://www.jstor.org/stable/1412159

[76] Michael S. Stinson, Lisa B. Elliot, and Ronald R. Kelly. 2017. Deaf and hard-of-hearing high school and college students' perceptions of speech-to-text and interpreting/note taking services and motivation. f. Dev. Phys. Disabil. 29, 1 (Feb. 2017), 131-152. DOI : https://doi.org/10.1007/s10882-017-9534-4

[77] Jessica J. Tran, Tressa W. Johnson, Joy Kim, Rafael Rodriguez, Sheri Yin, Eve A. Riskin, Richard E. Ladner, and Jacob O. Wobbrock. 2010. A web-based user survey for evaluating power saving strategies for deaf users of mobileASL. In Proceedings of the 12th International ACM SIGACCESS Conference on Computers and Accessibility (ASSETS'10). ACM, New York, NY, 115-122. DOI : https://doi.org/10.1145/1878803.1878825 
[78] Carol Bloomquist Traxler. 2000. The Stanford Achievement test: National norming and performance standards for deaf and hard-of-hearing students. f. Deaf Studies Deaf Educat. 5, 4 (2000), 337-348.

[79] John P. Wanous and Michael J. Hudy. 2001. Single-item reliability: A replication and extension. Organiz. Res. Methods 4, 4 (2001), 361-375. DOI : https://doi.org/10.1177/109442810144003

[80] John P. Wanous and Arnon E. Reichers. 1996. Estimating the reliability of a single-item measure. Psychol. Rep. 78, 2 (1996), 631-634. DOI : https://doi.org/10.2466/pr0.1996.78.2.631

[81] John P. Wanous, Arnon E. Reichers, and Michael J. Hudy. 1997. Overall job satisfaction: How good are single-item measures? J. Appl. Psychol. 82, 2 (1997), 247.

[82] World Federation of the Deaf. 2018. WFD and WASLI Statement on Use of Signing Avatars. Retrieved from https:// wfdeaf.org/news/resources/wfd-wasli-statement-use-signing-avatars/.

[83] Roger L. Worthington and Tiffany A. Whittaker. 2006. Scale development research. Counsel. Psychol. 34, 6 (Nov. 2006), 806-838. DOI : https://doi.org/10.1177/0011000006288127

[84] William R. Zwick and Wayne F. Velicer. 1982. Factors influencing four rules for determining the number of components to retain. Multivar. Behav. Res. 17, 2 (Apr. 1982), 253-269. DOI : https://doi.org/10.1207/s15327906mbr1702_5

Received April 2018; revised October 2018; accepted February 2019 